\title{
Experimental evaluation of low concentration scleroglucan biopolymer solution for enhanced oil recovery in carbonate
}

\author{
Vitor H.S. Ferreira*, and Rosangela B.Z.L. Moreno* \\ School of Mechanical Engineering, University of Campinas, Rua Mendeleyev, 200 - Cidade Universitária, Campinas, \\ SP 13083-860, Brazil
}

Received: 23 March 2020 / Accepted: 9 July 2020

\begin{abstract}
Injection of polymers is beneficial for Enhanced Oil Recovery (EOR) because it improves the mobility ratio between the displaced oil and the displacing injected water. Because of that benefit, polymer flooding improves sweep and displacing efficiencies when compared to waterflooding. Due to these advantages, polymer flooding has many successful applications in sandstone reservoirs. However, polymer flooding through carbonatic rock formations is challenging because of heterogeneity, high anionic polymer retention, low matrix permeability, and hardness of the formation water. The scleroglucan is a nonionic biopolymer with the potential to overcome some of those challenges, albeit its elevated price. Thus, the objective of this work is to characterize low concentration scleroglucan solutions focusing on EOR for offshore carbonate reservoirs. The laboratory evaluation consisted of rheology, filtration, and core flooding studies, using high salinity multi-ionic brines and light mineral oil. The tests were run at $60^{\circ} \mathrm{C}$, and Indiana limestone was used as a surrogate reservoir rock. A rheological evaluation was done in a rotational rheometer aiming to select a target polymer concentration for the injection fluid. Different filtration procedures were performed using membrane filters to prepare the polymer solution for the displacement process. Core flooding studies were done to characterize the polymer solution and evaluate its oil recovery relative to waterflooding. The polymer was characterized for its retention, inaccessible pore volume, resistance factor, in-situ viscosity, and permeability reduction. Rheology studies for various polymer concentrations indicated a target scleroglucan concentration of $500 \mathrm{ppm}$ for the injection solution. Among the tested filtration methods, the best results were achieved when a multi-stage filtration was performed after an aging period of $24 \mathrm{~h}$ at $90{ }^{\circ} \mathrm{C}$ temperature. The single-phase core flooding experiment resulted in low polymer retention $(20.8 \mu \mathrm{g} / \mathrm{g})$, inaccessible pore volume (4.4\%), and permeability reduction (between 1.7 and 2.4). The polymer solution in-situ viscosity was slightly lower and less shear-thinning than the bulk one. The tested polymer solution was able to enhance the oil recovery relative to waterflooding, even with a small reduction of the mobility ratio ( $38 \%$ relative reduction). The observed advantages consisted of water phase breakthrough delay ( $172 \%$ relative delay), oil recovery anticipation $(159 \%$ and $10 \%$ relative increase at displacing fluid breakthrough and $95 \%$ water cut, respectively), ultimate oil recovery increase $(6.3 \%)$, and water-oil ratio reduction ( $38 \%$ relative decrease at $95 \%$ water cut). Our results indicate that the usage of low concentration scleroglucan solutions is promising for EOR in offshore carbonate reservoirs. That was supported mainly by the low polymer retention, injected solution viscosity maintenance under harsh conditions, and oil recovery anticipation.
\end{abstract}

\section{Nomenclature}

BPR Back Pressure Regulator

C Cannela's tuning constant

c Concentration

DI Deionized

EOR Enhanced Oil Recovery

FR Filtration Ratio
HPAM Partially Hydrolyzed Polyacrylamide

IAPV Inaccessible Pore Volume

$K \quad$ Power law fluid flow consistency index

$k \quad$ Absolute permeability

$k_{\mathrm{p}} \quad$ Effective permeability of phase "p"

$m \quad$ Mass

$N_{\mathrm{p}} \quad$ Cumulative produced oil

$n \quad$ Power law fluid flow behavior index

\footnotetext{
* Corresponding authors: vhsf@unicamp.br, zanoni@fem.unicam.br
} 


$\begin{array}{ll}\text { ORF } & \text { Oil Recovery Factor } \\ Q & \text { Volumetric flow rate } \\ \mathrm{RF} & \text { Resistance Factor } \\ \mathrm{RRF} & \text { Residual Resistance Factor } \\ \text { SCLG } & \text { Scleroglucan } \\ s_{\mathrm{p}} & \text { Saturation of phase "p" } \\ t & \text { Time } \\ \text { TDS } & \text { Total Dissolved Solids } \\ V & \text { Volume } \\ \text { VL } & \text { Viscosity Loss } \\ \text { VPI } & \text { Injected Pore Volume } \\ \text { WOR } & \text { Cumulative Water-Oil Ratio } \\ \mathrm{W}_{\mathrm{p}} & \text { Cumulative produced water } \\ \Gamma & \text { Retention } \\ \dot{\gamma} & \text { Shear rate } \\ \Delta p & \text { Pressure differential } \\ \eta & \text { Apparent viscosity } \\ \Phi & \text { Porosity } \\ \mu & \text { Viscosity } \\ v & \text { Superficial or Darcy velocity } \\ \rho & \text { Density }\end{array}$

\section{Subscripts}

$\begin{array}{ll}\text { is } & \text { In }- \text { situ } \\ \text { OOIP } & \text { Original Oil In Place } \\ \text { o } & \text { Oil phase } \\ \mathrm{p} & \text { Polymer component } \\ \mathrm{s} & \text { Salt component } \\ \mathrm{w} & \text { Water phase }\end{array}$

\section{Superscripts}

$\begin{array}{ll}\text { 1st } & \text { First polymer injection } \\ \text { 2nd } & \text { Second polymer injection } \\ \text { after } & \text { After the porous medium has contact } \\ & \text { with polymer } \\ \text { before } & \begin{array}{l}\text { Before the porous medium has contact } \\ \text { with polymer }\end{array} \\ \text { ref } & \text { Reference value }\end{array}$

\section{Introduction}

Polymer flooding is an Enhanced Oil Recovery (EOR) method based on the addition of water-soluble polymers to the injection water. These polymer solutions result in increased viscosity and reduced effective permeability of the displacing fluid, thus, reducing its mobility (Taber and Martin, 1983). This mobility control capability improves the oil sweep, which in turn leads to oil recovery anticipation and water management improvement relative to waterflooding (Sorbie, 1991). Some authors have recommended polymer flooding to mitigate viscous fingerings in reservoirs containing highly viscous oils (Delamaide et al., 2014; Kang et al., 2011), or to control channeling effects in heterogeneous reservoirs (Demin et al., 1996; Liu et al., 2007). This technique has a history of field implementations for decades with many successful cases, albeit mostly in sandstone reservoirs (Standnes and Skjevrak, 2014).

Carbonate reservoirs account for around $50 \%$ to $60 \%$ of the world's total oil and gas reserves (Sha et al., 2019; Zaeri et al., 2018). However, these reservoirs are characterized by heterogeneities on different scales (He et al., 2014; Sha et al., 2019; Wei et al., 2015; Ziauddin and Bize, 2007) and a tendency to oil wetness (Al-Hadhrami and Blunt, 2000; Hirasaki and Zhang, 2004; Wardlaw, 1996; Zaeri et al., 2018). These characteristics difficult the oil recovery in such reservoirs. Polymer flooding is an option to overcome heterogeneity issues, but the technique presents additional challenges associated with some carbonate reservoirs such as high reservoir temperature and high salinity/ hardness injection and formation water. These characteristics lead authors to discourage the implementation of polymer floods in naturally fractured carbonates (Al-Adasani and Bai, 2010; Boekhout, 2015; Bourdarot and Ghedan, 2011; Carcoana, 1982; Chang, 1978; Kang et al., 2016; Littmann, 1988; de Melo et al., 2002; Meyer et al., 2007; Sorbie, 1991; Taber et al., 1997).

According to a survey reported by Sheng et al. (2015) containing 733 polymer flooding field projects, only $1 \%$ were offshore, and less than $15 \%$ were in carbonates. A similar report by Standnes and Skjevrak (2014) containing 72 field applications of polymer flooding revealed that $8 \%$ were offshore and $7 \%$ in carbonates, with no offshore projects in carbonate formations. The use of traditional synthetic polymers justifies those few applications in offshore carbonate reservoirs, since Hydrolyzed Polyacrylamide (HPAM) is sensitive to temperature, brine hardness, and presents high adsorption in carbonates (Ghannam and Esmail, 1998; Kamal et al., 2015; Methemitis et al., 1986; Moradi-Araghi and Doe, 1987; Xin et al., 2008).

Biopolymers are polymers produced from living organisms, e.g., bacteria, fungi, plants. Some biopolymers have desirable characteristics to a variety of processes, being used in food, pharmaceutical, cosmetics, paint, textile, construction, and oil industries, among others (El-Haddad, 2014; Gao, 2016; Pu et al., 2017; Roy et al., 2009; Thombare et al., 2016; Zhong et al., 2013). The advantages of biopolymers targeted by the oil industry over synthetic ones are their eco-friendliness, superior chemical and mechanical stabilities, and tolerance to high temperature and salinity (Bourdarot and Ghedan, 2011; Pu et al., 2017). The main drawbacks of biopolymers relative to synthetic polymers are their relatively higher cost, susceptibility to biological degradation, and non-polymer debris presence due to their extraction process ( $\mathrm{Pu}$ et al., 2017). The last of which can result in poor filterability and lead to formation damage (Fournier et al., 2018; Rivenq et al., 1992). Considering their advantages, biopolymers are promising for EOR application, especially considering offshore reservoirs (Bourdarot and Ghedan, 2011).

The biopolymer Scleroglucan (SCLG) is produced by fermentation of a plant pathogen fungus of the genus 


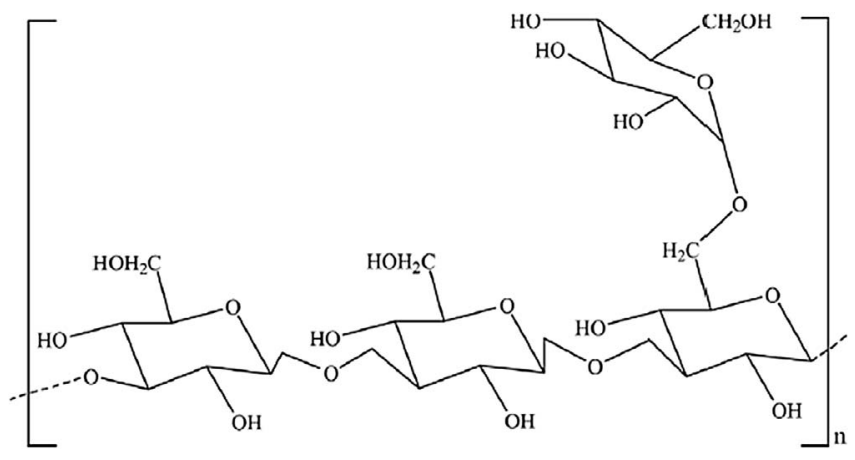

Fig. 1. Chemical structure of the scleroglucan (adapted from $\mathrm{Pu}$ et al., 2017).

Sclerotium (Rivenq et al., 1992). The SCLG is a non-ionic polysaccharide that assumes a rigid triple helix structure in solution (Yanaki et al., 1981). The chemical composition of the scleroglucan is shown in Figure 1 and is the same as the biopolymer Schizophyllan (Rivenq et al., 1992).

The scleroglucan polymer confers high viscosity to solutions for small amounts of polymer, has excellent shear resistance and temperature stability ( $\mathrm{Pu}$ et al., 2017), and is very resistant to salt composition (Gallino et al., 1996; Rivenq et al., 1992) and pH (Fariña et al., 2001). Scleroglucan is a non-ionic polymer; therefore, its adsorption in carbonates is lower than the one experienced by HPAM. HPAM is anionic, and because of that, it presents high adsorption in carbonate rocks due to the strong interaction between $\mathrm{Ca}^{2+}$, in the rock surface, and $\mathrm{COO}^{-}$, in the polymer backbone (Kamal et al., 2015). Thus, the SCLG biopolymer is a promising candidate for the EOR application in carbonates.

According to Seright (2017), polymer concentrations typically injected for EOR have progressively increased from the 1970 s to present, with more recent projects injecting close to $1000 \mathrm{ppm}$ or more of polymer concentration. However, these projects have used mostly HPAM, which have considerable drawbacks for offshore application in carbonate formations, as previously mentioned. Thus, in light of the relatively high cost of the scleroglucan biopolymer, if a lower concentration is required to reach the same target viscosity designed for HPAM, the biopolymer flooding can be cost-effective for those fields where HPAM is not technically adequate.

\subsection{Relevant polymer characteristics for EOR applications}

Polymer solutions are complex fluids, and many characteristic phenomena are associated with their displacement through porous media. That makes difficult the prediction of their behavior and requires laboratory analyses.

Polymer solutions are known to be non-Newtonian fluids, i.e., the viscosity is dependent on the shear rate experienced by the liquid. More specifically, polymer solutions are shear-thinning (or pseudoplastic); that is, the solution viscosity decreases as the shear rate increases (Sorbie, 1991). That occurs because the large polymer molecules tend to align themselves with the flow field (Padsalgikar, 2017).

However, the apparent viscosity exhibited by polymer solutions when flowing through porous media is different from that measured in rheometers/viscometers (Chauveteau and Zaitoun, 1981; Stavland et al., 2010). This difference is mainly due to the complexity of the pore network (Sorbie, 1991). According to the literature, rigid polymers or flexible ones under low shear rate exhibit in-situ viscosity lower than the bulk one. Some authors interpret that this phenomenon occurs because the polymer does not flow through the entire pore space during its transport (Chauveteau and Zaitoun, 1981; Chauveteau et al., 1984; Sorbie, 1990; Stavland et al., 2010). Flexible polymers at high shear rates exhibit a shear thickening behavior in-situ not observed in bulk viscosity measurements, which is associated with the viscoelastic properties of such polymers (Heemskerk et al., 1984; Jennings et al., 1971; Seright et al., 2009; Stavland et al., 2010).

As the polymer flows through a porous medium, several interactions between the medium and the polymer can lead to polymer retention (Sorbie, 1991). The retention mechanisms strip polymer from the injected solution, leading to the formation of an injection fluid bank partially or completely polymer-free (Sorbie, 1991). Polymer retention refers to the mechanisms of adsorption, mechanical entrapment, and hydrodynamic retention (Sheng, 2011). Adsorption is governed by weak intermolecular forces and can be considered an instantaneous and irreversible phenomenon (Goddard and Gruber, 1999; Ruthven, 1984; Zhang and Seright, 2014). Mechanical entrapment occurs in porous media restrictions too small relative to the polymer molecular size (Huh et al., 1990; Willhite and Dominguez, 1977) and, if the solution is screened and processed correctly, its contribution to overall retention is minimal (Sorbie, 1991). Hydrodynamic retention is flow rate-dependent and reversible, resulting from molecule-trapping in flow-stagnant zones, and is a minor contributor to overall retention (Chauveteau and Lecourtier, 1988; Sorbie, 1991; Willhite and Dominguez, 1977; Zhang and Seright, 2015).

One can observe a permanent permeability reduction when a polymer flows through porous media (Sorbie, 1991). This phenomenon is also known as the Residual Resistance Factor (RRF). One can interpret its effect as a reduction in the effective pore diameter, which can be caused by polymer adsorption or mechanical entrapment (Baijal and Dey, 1982; Dupuis et al., 2011). The RRF can be relevant even when small amounts of a polymer are retained (Jennings et al., 1971).

In the absence of retention, the polymer molecules are transported through porous media faster than small molecules (e.g., salts), a phenomenon known as Inaccessible Pore Volume (IAPV) (Dawson and Lantz, 1972; Sorbie, 1990). One can interpret the IAPV as the result of pore size incompatibility (Dawson and Lantz, 1972) or depleted layer effects (Sorbie, 1990). The first effect occurs on pores too small compared to the polymer molecular size or blocked pores, forcing the polymer to flow only through large enough channels (Dawson and Lantz, 1972). The depleted layer effect is a consequence of a thin layer of polymer-free 
fluid near the pore surfaces, meaning that the polymer does not flow through the entire pore volume (Sorbie, 1990).

Dispersion, viscoelasticity, and degradation mechanisms are other phenomena important for EOR applications, but they are not within the scope of this work.

\subsection{Objective}

This paper aims to evaluate scleroglucan flooding for EOR in offshore carbonate reservoirs while exploring a low polymer concentration. The relatively high cost of the scleroglucan balanced by its high viscosifying power and high resistance to temperature and brine composition motivates the study. The evaluation consists of rheology, filtration, and core flooding experiments to assess polymer solution filterability, transport properties in porous media, rock-fluid interactions, and oil recovery performance relative to waterflooding.

\section{Materials and methods}

The experimental evaluation of scleroglucan consisted of rheological characterization, filtration studies, and core flooding tests. Notably, two types of core flooding experiments were performed, single-phase and two-phase. The single-phase experiment aimed to evaluate polymer adsorption, IAPV, RRF, and in-situ viscosity. The two-phase test focused on the comparative performance of oil recovery by polymer flooding against waterflooding, as well as on the validation of RRF and in-situ viscosity estimates in the presence of oil.

\subsection{Fluid preparation and characterization}

We used four fluids in the experiments: two brines, a polymeric solution, and white mineral oil. The compositions of the two brines are reported in Table 1.

Brine A was used as a solvent to the polymeric solution and as brine in the two-phase core flooding experiment. Brine B was used in the single-phase test as it contrasts with the polymer solution salinity.

The polymer used in this work was a non-EOR-grade scleroglucan by Carbosynth. The polymer was supplied in powder form and with purity higher than $90 \%$, according to the supplier. A stock polymeric solution was first prepared considering $4000 \mathrm{ppm}$ of scleroglucan in Deionized (DI) water to favor chain hydration (Beall et al., 2004). Before preparing the solution, the DI water and the polymer were weighted in separate beakers. The DI water was put on a magnetic stirrer with the vortex adjusted to $90 \%$ of the liquid height; then, the polymer powder was sprinkled on the shoulders of the vortex. The time to sprinkle all the polymer in the solution was between 20 and $30 \mathrm{~min}$ to avoid the formation of agglomerates. After all the polymer was put in the DI water, the liquid was covered with plastic film, and the system was kept under high agitation for 10 days. After this period of agitation, the stock solution preparation process was complete. This stock solution was then diluted to achieve the target polymer concentration in $100 \%$ Brine A. Note that, to achieve the target polymer concentration in 100\% Brine A, the stock solution was diluted in a concentrated brine. Each dilution remained stirring overnight to homogenize the solution.

After the preparation of the fluids, we characterized them in terms of viscosity, density and $\mathrm{pH}$. The density was acquired in a densimeter based on the pulsed excitation method. The viscosity was measured in a rotational rheometer using a single gap concentric cylinders geometry. The flow curves were obtained by constant rate measurements for each shear rate, waiting $30 \mathrm{~s}$ between setting the shear rate and performing the viscosity measurement to ensure that the system was under steady-state conditions. The basic characteristics of the fluids are summarized in Table 2 and Figure 2.

\subsection{Filtration experiments}

After diluting the polymer stock solution to obtain $1000 \mathrm{ppm}$ of SCLG in Brine A, we filtered them using mixed cellulose esters membranes known commercially as MF-Millipore. The filters had $142 \mathrm{~mm}$ of diameter and, according to the supplier, were hydrophilic. The meshes tested were $8 \mu \mathrm{m}$, and $1.2 \mu \mathrm{m}$. The $1.2 \mu \mathrm{m}$ mesh is used by many authors considering polymer applications in EOR (Kulawardana et al., 2012; Rivenq et al., 1992).

We filtered the solutions according to the recommendations in API RP 63 (API, 1990). Firstly, we saturated the filter with DI water and the capillary tubes with the polymer solution. Then, we pressurized the system with nitrogen at 30 psi and opened the outlet valve to allow filtration. We collected the filtered solution in graduated cylinders and recorded the filtered volume over time. We discarded the first $60 \mathrm{~cm}^{3}$ of the filtered solution as it might be mixed with the DI water used for the filter saturation.

We studied the filtration process by using a single filter, or multiple filtrations using successively finer filters. In the first method, only one filtration was considered, while in the second method, we first filtered the solution in the $8 \mu \mathrm{m}$ mesh filter, followed by the filtration of the collected samples through the finer $1.2 \mu \mathrm{m}$ filter.

We also evaluated the filtration considering the inclusion and the absence of a technique for filterability improvement. We filtered both fresh polymer solutions, and solutions which went through a filterability improvement procedure based on the works of Rivenq et al. (1992) and Kulawardana et al. (2012). Such technique consisted of aging the scleroglucan solutions for $24 \mathrm{~h}$ under $90^{\circ} \mathrm{C}$ inside a stainless steel container. After placing the polymer solution in the fluid bottle, we bubbled nitrogen gas through the solution to mitigate oxidative degradation. This aging process aims at removing polymer aggregates within the solution.

We evaluated the filterability through the Filtration Ratio (FR), equation (1), as well as by the Viscosity Loss (VL) after filtration, equation (2): 
Table 1. Compositions of the brines.

\begin{tabular}{|c|c|c|}
\hline \multirow[t]{2}{*}{ Salt } & \multicolumn{2}{|c|}{ Concentration (ppm) } \\
\hline & Brine A & Brine B \\
\hline $\mathrm{KCl}$ & 749.3 & 374.7 \\
\hline $\mathrm{MgCl}_{2} \cdot 6 \mathrm{H}_{2} \mathrm{O}$ & 1271.3 & 635.7 \\
\hline $\mathrm{SrCl}_{2} \cdot 6 \mathrm{H}_{2} \mathrm{O}$ & 5.2 & 2.6 \\
\hline $\mathrm{LiCl}$ & 1.2 & 0.6 \\
\hline $\mathrm{NaBr}$ & 82.4 & 41.2 \\
\hline $\mathrm{Na}_{2} \mathrm{SO}_{4}$ & 57.7 & 28.8 \\
\hline $\mathrm{NaCl}$ & 28251.3 & 14125.7 \\
\hline
\end{tabular}

Table 2. Density, viscosity, and $\mathrm{pH}$ of the solutions at $60^{\circ} \mathrm{C}$.

\begin{tabular}{lcccc}
\hline Fluid & Oil & Brine A & Brine B & Polymer solution \\
\hline Viscosity $^{*}$ & $6.65 \pm 0.09$ & $0.545 \pm 0.016$ & $0.523 \pm 0.027$ & Figure 2 \\
Density $^{*}$ & $0.81857 \pm 0.00001$ & $1.00592 \pm 0.00001$ & $0.99369 \pm 0.00001$ & $1.00692 \pm 0.00001$ \\
$\mathrm{pH}^{\dagger}$ & - & $5.39 \pm 0.01$ & $5.74 \pm 0.01$ & $5.53 \pm 0.01$ \\
\hline
\end{tabular}

* Measured at $60{ }^{\circ} \mathrm{C}$ and ambient pressure.

$\dagger$ Measured at ambient temperature and pressure.
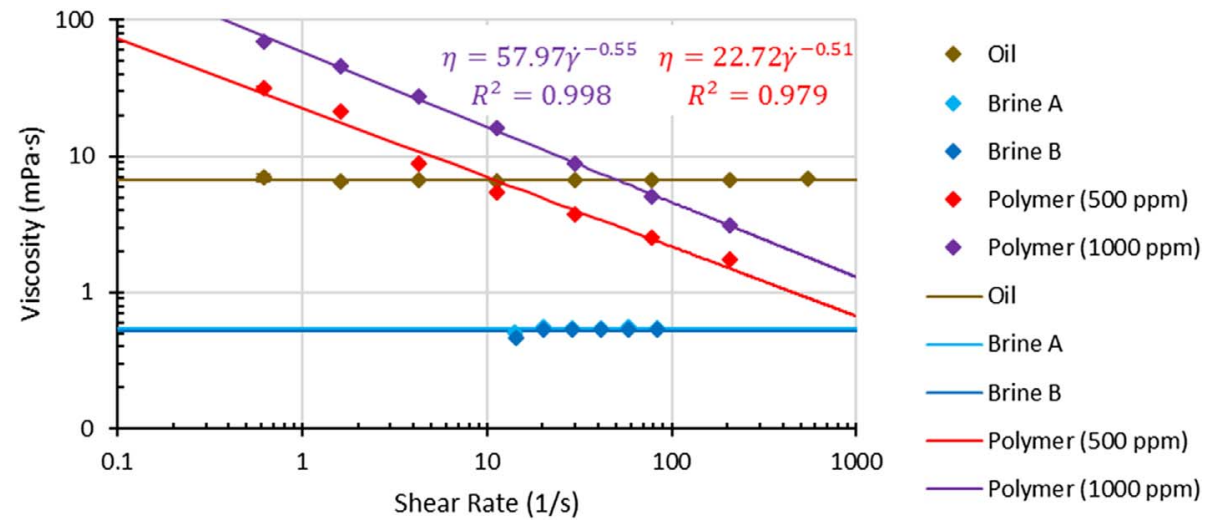

Fig. 2. Viscosity of the fluids used in the tests at $60^{\circ} \mathrm{C}$. Note that the polymer viscosity reported in this figure corresponds to the one used in the core flooding experiments, i.e., after the filtration process.

$$
\begin{gathered}
\mathrm{FR}_{V_{4}}=\frac{t_{V_{4}}-t_{V_{3}}}{t_{V_{2}}-t_{V_{1}}}, \\
\mathrm{VL}=\frac{\eta_{\mathrm{p}}^{\mathrm{ref}}-\eta_{\mathrm{p}}}{\eta_{\mathrm{p}}^{\mathrm{ref}}},
\end{gathered}
$$

where $t_{V}$ is the time necessary to filtrate the volume $V$ of solution, and $\eta_{\mathrm{p}}$ is the polymer solution apparent viscosity. The parameters $V_{4}$ and $V_{3}$ correspond to the volumes close to the end of the filtration process, while $V_{2}$ and $V_{1}$ represent the volumes collected at the start of the process. Note that $V_{4}-V_{3}=V_{2}-V_{1}$. The superscript ref corresponds to a reference viscosity value before the filtration.

\subsection{Core handling and characterization}

We used Indiana limestone for the core flooding experiments. The basic petrophysical properties of the cores are presented in Table 3.

We measured the porosity informed in Table 3 by gas porosimetry, and the permeability by brine injection and pressure measurement in the first steps of each core flooding experiment. 
Table 3. Characteristics of the cores.

\begin{tabular}{lccc}
\hline Property & Unit & \multicolumn{3}{c}{ Value } \\
\hline Identification & - & IL00 & IL09 \\
Experiment & - & Single-phase core flooding & Two-phase core flooding \\
Dry mass & $\mathrm{g}$ & $500.8 \pm 0.1$ & $763.1 \pm 0.1$ \\
Length & $\mathrm{cm}$ & $19.90 \pm 0.05$ & $29.90 \pm 0.05$ \\
Diameter & $\mathrm{cm}$ & $3.780 \pm 0.005$ & $3.810 \pm 0.005$ \\
Porosity & - & $0.157 \pm 0.004$ & $0.160 \pm 0.004$ \\
Permeability & $\mathrm{mD}$ & $373 \pm 10$ & $336 \pm 4$ \\
\hline
\end{tabular}

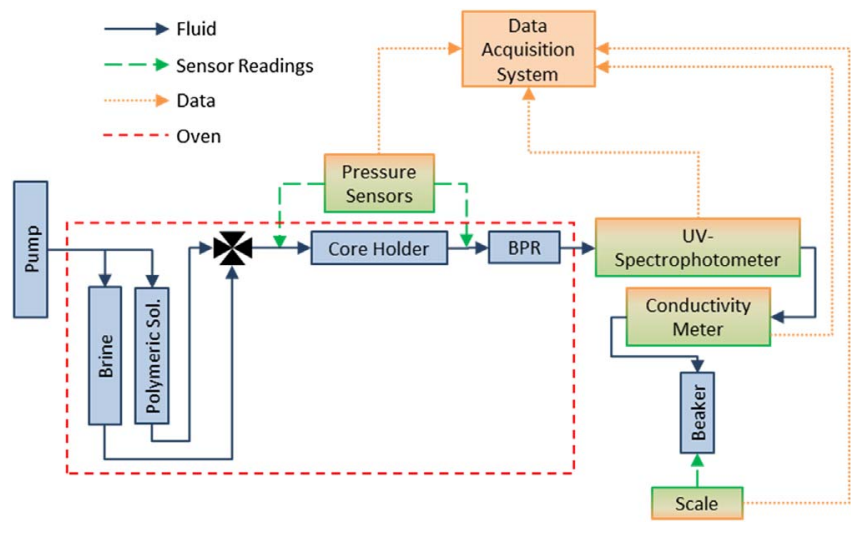

Fig. 3. Single-phase core flooding experimental setup.

The core was cleaned with methanol and toluene by soxhlet extraction, as recommended by API RP 40 (API, 1998).

\subsection{Single-phase core flooding}

The single-phase core flooding used two fluids: brine (Brine B) and polymer solution. It aimed to characterize polymer retention, IAPV, permeability reduction, Resistance Factor (RF) and in-situ viscosity.

\subsubsection{Equipment}

Figure 3 depicts the experimental setup.

A syringe pump (error: $0.1 \%$ of set point) injects distilled water into the fluid accumulators. The accumulators are bottles made of stainless steel with floating pistons to allow injection without mixing the distilled water with brine/polymeric solution. A valve assembly selects which fluid is injected into the core. A core holder confines the rock core inside a rubber sleeve, and a Back-Pressure Regulator (BPR) positioned at the outlet of the core holder controls the pore pressure inside the rock sample. After the BPR, there are an ultraviolet spectrophotometer (error: $3 \%$ of measurement) and a conductivity meter (error: $4 \%$ of measurement), which measure the polymer and salt (tracer) concentrations, respectively. We used a wavelength of $220 \mathrm{~nm}$ to measure the polymer, as wavelengths of this range can detect carbohydrates (Kaijanen et al., 2015; Pereira da Costa and Conte-Junior, 2015). A beaker placed on top of a scale (error: $0.1 \mathrm{~g}$ ) collects the production fluids after they pass through the equipment mentioned above. A series of differential pressure sensors are positioned in the pressure-tapped core holder in order to measure the pressure drop along the flow direction. Each pressure sensor measured the pressure drop relative to the production face of the core, by hydraulically connecting the low-pressure side of the sensors to the outlet core holder diffuser. We positioned three pressure taps at $6.35,11.43$, and $16.51 \mathrm{~cm}$ from the injection face, or $13.55,8.47$, and $3.39 \mathrm{~cm}$ from the production face. Data acquisition and logging systems record all the data.

We performed the tests under $60{ }^{\circ} \mathrm{C}$ and 2000 psi pore pressure (i.e., 2000 psi of back pressure).

\subsubsection{Experimental procedure}

Figure 4 shows the experimental sequence of the singlephase core flooding. The brine injections refer to the Brine $\mathrm{B}$, while the polymer ones indicate a filtered $500 \mathrm{ppm}$ SCLG solution in Brine A.

The sample saturation with brine was done by confining the core inside the core holder, and then submitting it to vacuum pressure. After under vacuum for a couple of hours, the brine was slowly admitted to the core until we observed its breakthrough in the production side.

The first brine injection aimed to clean the core of any fines and determine the absolute permeability to water of the sample. We injected 45 pore volumes of brine for this cleanup. We determine the absolute permeability to water through Darcy's Law for linear displacements, using five different flow rates in this step: $0.25,0.5,1.0,1.5$ and $2.0 \mathrm{~cm}^{3} / \mathrm{min}$.

Figure 5 shows the injected pore volume and the injection volumetric flow rate for each subsequent brine and polymer injections.

During the second polymer injection, the multi-rate floodings aimed to allow estimation of RF and in-situ viscosities for multiple flow velocities and shear rates.

Note that the polymer injections to displace water consisted of at least 20 pore volumes, while the water injections to displace polymer were of at least 50 pore volumes. These large volumes were employed to guarantee steady-state conditions. We changed the fluid or the flow rate only after the system reached steady-state conditions. That was verified by analyzing pressure drops and produced polymer/salt 


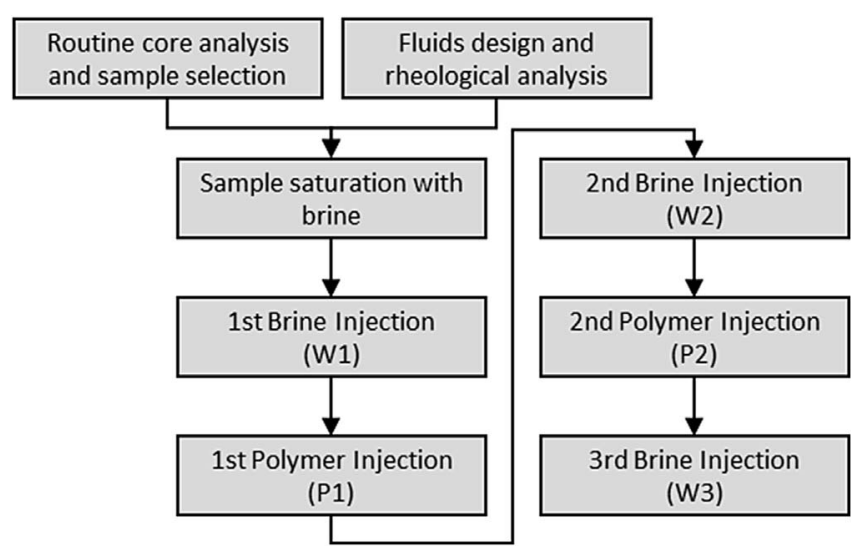

Fig. 4. Single-phase core flooding sequence.

concentrations. We considered the steady-state regime when the pressure reading was stable for several hours, and the produced polymer and salt concentrations were equal to the injected ones.

The mobility reduction (or Resistance Factor - RF) is estimated by equation (3), while applying equation (4), we can calculate the permeability reduction (or RRF):

$$
\begin{gathered}
\mathrm{RF}=\frac{\Delta p_{\mathrm{p}}}{\Delta p_{\mathrm{w}}^{\text {before }}}, \\
\mathrm{RRF}=\frac{\Delta p_{\mathrm{w}}^{\text {after }}}{\Delta p_{\mathrm{w}}^{\text {before }}},
\end{gathered}
$$

where $\Delta p$ is the pressure drop across the core, the subscripts $p$ and $w$ indicate polymer and brine injections respectively, and the superscripts before and after correspond to the measurement done before the first polymer injection and after each polymer injection, respectively. We took all the pressure measurements after steady-state conditions.

With both RF and RRF at hand, one can estimate the polymer in-situ viscosity $\left(\eta_{\mathrm{p}, \text { is }}\right)$ using equation (5):

$$
\eta_{p, i s}=\frac{\mathrm{RF}}{\mathrm{RRF}} \cdot \mu_{\mathrm{w}}
$$

where $\mu_{\mathrm{w}}$ is the brine viscosity.

We used Cannella's equation to estimate the in-situ shear rate $\left(\dot{\gamma}_{\text {is }}\right)$, as in equation (5) (Cannella et al., 1988):

$$
\dot{\gamma}_{\text {is }}=C \cdot\left[\frac{3 \cdot n+1}{4 \cdot n}\right]^{\frac{n}{n-1}} \cdot \frac{v}{\sqrt{k_{w} \cdot s_{w} \cdot \phi}}
$$

where $\varphi$ is the porosity of the core, $s_{\mathrm{w}}$ is the water saturation, $k_{\mathrm{w}}$ is the effective water permeability, $v$ is the superficial (or Darcy) velocity, $n$ is the Power-law fluid flow behavior index, and $C$ is the Cannella tuning constant, generally assumed to be equal to 6 (Cannella et al., 1988; Sorbie, 1991). Note that, for a single-phase experiment, the $k_{\mathrm{w}}$ is equal to the absolute permeability, and the $s_{\mathrm{w}}$ is equal to 1 .

We used the double polymer bank method, proposed by Lotsch et al. (1985), to estimate the polymer retention. In this method, we use equation (7) to estimate the polymer retention $\left(\Gamma_{\mathrm{p}}\right)$ :

$$
\Gamma_{\mathrm{p}}=\frac{\left(m_{\mathrm{p}}^{\mathrm{P} 2}-m_{\mathrm{p}}^{\mathrm{P} 1}\right)}{m_{\mathrm{r}}}
$$

where $m_{\mathrm{r}}$ is the bulk dry core mass and $m_{\mathrm{p}}$ is the produced polymer mass, estimated by equation (8):

$$
m_{\mathrm{p}}=\int_{t_{\mathrm{start}}}^{t_{\mathrm{end}}} c_{\mathrm{p}} \cdot Q \cdot \rho_{\mathrm{p}, \mathrm{sol}} \cdot \mathrm{d} t,
$$

where $c_{\mathrm{p}}$ is the polymer concentration in the effluent, $Q$ is the volumetric flow rate, $\rho_{\mathrm{p}, \mathrm{sol}}$ is the polymer solution density, and $t$ is the time. The time subscripts refer to the start and the end of the injection. Note that we consider the end of the second polymer injection before any flow rate change, therefore the sections considered for retention estimation were identical in terms of injection. We verified that the produced polymer concentration had reached the same value as the injected one by $t_{\text {end }}$.

We determined the IAPV by the method proposed by Dawson and Lantz (1972), which can be expressed by equation (9):

$$
\operatorname{IAPV}_{\mathrm{p}}=\operatorname{VPI}\left(c_{\mathrm{p}, \text { tres }}^{\mathrm{P} 2}\right)-\operatorname{VPI}\left(c_{\mathrm{s}, \text { tres }}^{\mathrm{P}}\right)
$$

where VPI indicates the injected pore volume correspondent to the threshold concentrations $c_{\mathrm{p} \text {,tres }}$ and $c_{\mathrm{s} \text {,tres }}$, for the polymer and salt, respectively. The target concentrations of this experiment were the average concentrations between brine and polymer solutions, i.e., $250 \mathrm{ppm}$ of polymer and the average salt concentration between brines $\mathrm{A}$ and B (see Tab. 1).

Note that the IAPV is measured in the second polymer injection because the retention is satisfied in the first polymer injection, which means that no retention takes place during IAPV calculation.

\subsection{Two-phase core flooding}

The two-phase core flooding uses three fluids: oil, brine (Brine A), and polymer solution. This experiment aims to investigate the oil recovery by polymer flooding versus waterflooding, as well as validating RRF and in-situ viscosity estimates in the presence of oil.

\subsubsection{Equipment}

The experimental setup is shown in Figure 6 .

The inlet side of this experimental setup is very similar to the single-phase one, with only the addition of an oil accumulator. This accumulator was also made of stainless steel, but it had not a floating piston, as the gravity and non-miscibility of water and oil guaranteed the oil being pushed to the core when selected by the valve. One the other hand, production equipment differs from the singleto two-phase experiments. For the two-phase experiment, there are a series of valves, two-separators, and a back pressure regulator valve. These valves direct the fluid to the top or the bottom of the separator or through a separator bypass. These valves are essential to accumulate water (bottom-entry, top-exit) or oil (top-entry, bottom-exit) into the separators, depending on the cumulative volumes to be 

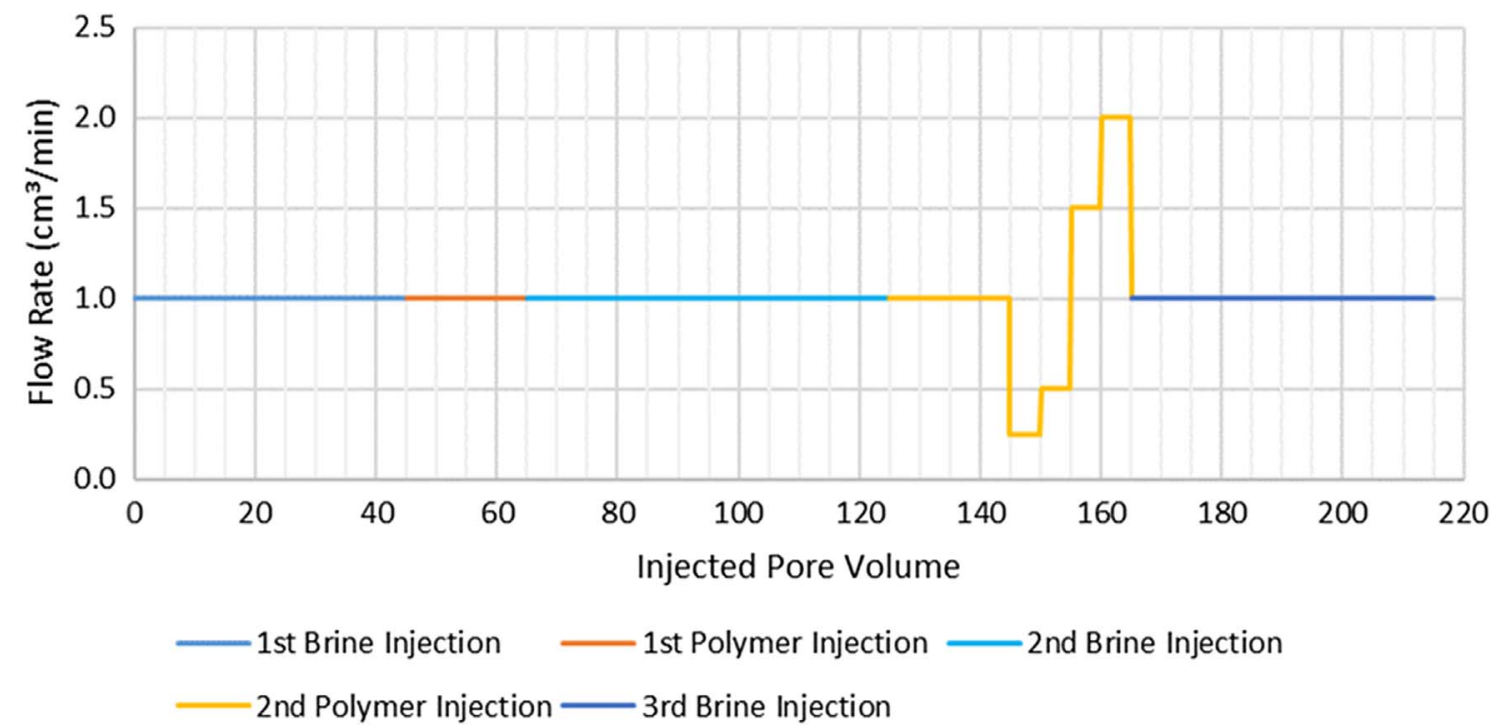

Fig. 5. Volumetric injection flow rate versus injected pore volume for each brine and polymer injection shown in Figure 4 .

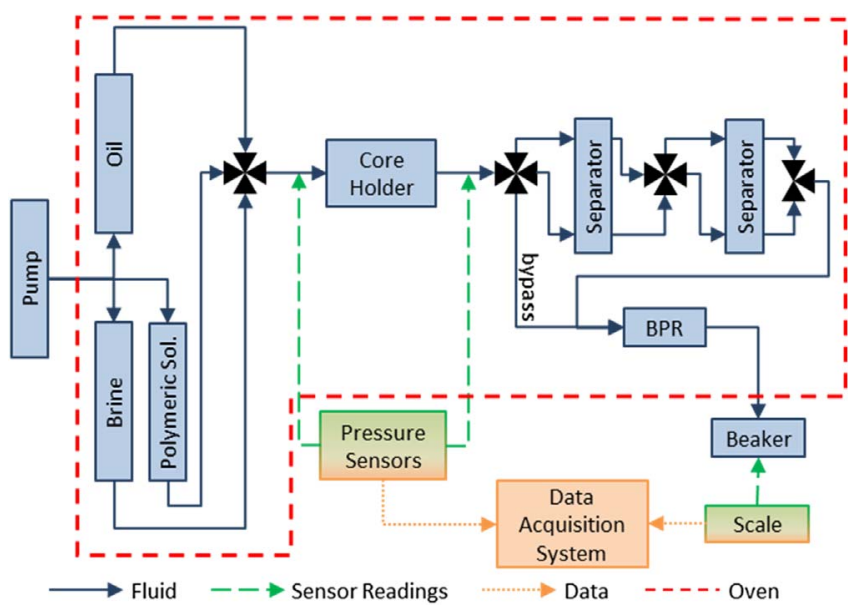

Fig. 6. Two-phase core flooding experimental setup.

read. After the fluids pass the back-pressure regulator, they are produced in a beaker, on top of a scale.

Similar to the single-phase experiments, we mounted the pressure sensor to read the pressure drop relative to the production face. We had three pressure taps, which were positioned at $11.43,16.51$, and $21.59 \mathrm{~cm}$ from the injection face, or $18.47,13.39$, and $8.31 \mathrm{~cm}$ from the production face.

The experiment was performed under $60{ }^{\circ} \mathrm{C}$ and 3000 psi of back pressure.

\subsubsection{Experimental procedure}

The experimental sequence for the two-phase core flooding is presented in Figure 7. The water injections refer to the Brine A, while the polymer one refers to a filtered 500 ppm SCLG solution in Brine A.

The core saturation and absolute permeability measurements in the two-phase core flooding experiment were identical to the single-phase one, with the exception of the flow rates in which the absolute permeability was measured, as we used $0.25,0.5,0.75,1$, and $2 \mathrm{~cm}^{3} /$ min. During this step, the production was carried on through the bypass shown in Figure 6, as the separators start initially saturated with oil.

All the injections after the measurement of absolute permeability were performed at $0.5 \mathrm{~cm}^{3} / \mathrm{min}$. The injections O1, WF, O2, PF, and WPF corresponded to 15, 25, 22.6, 27.7, and 17 injected pore volumes, respectively. During the injection of aqueous fluids, the cumulative produced oil was monitored in the separators, while for the oil injections, the separators monitored the cumulative produced water. This production monitoring could be done by adjusting the valve assemblies in the separators.

By the end of each injection, we estimated the terminal saturation (irreducible water for oil injections, and residual oil for water injections) by mass balance, and the effective permeability by Darcy's law. The permeability measured in the water post-flooding was considered to be the same for the polymer flooding.

During the WF and PF steps, we estimated the Oil Recovery Factor (ORF) and cumulative Water-Oil Ratio (WOR), as defined by equation (10) and equation (11), respectively:

$$
\begin{gathered}
\text { ORF }=\frac{N_{\mathrm{p}}}{V_{\text {OOIP }}}, \\
\text { WOR }=\frac{W_{\mathrm{p}}}{N_{\mathrm{p}}},
\end{gathered}
$$

where $N_{\mathrm{p}}$ is the cumulative produced oil during a target injection, $V_{\text {OOIP }}$ is the original oil volume in place at the start of the same target injection, $W_{\mathrm{P}}$ is the cumulative produced water during the same target injection.

The RF, RRF, polymer in-situ viscosity and shear rate were determined through equations (3)-(6), respectively. 


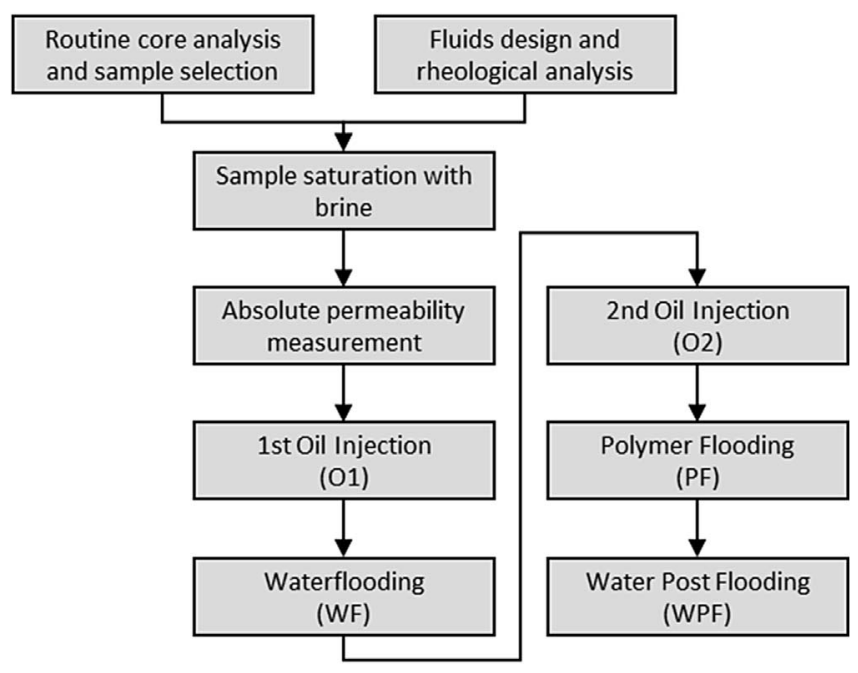

Fig. 7. Two-phase core flooding sequence.

Note that the pressure measurement labeled as "before" is taken in the WF step, and that one marked as "after" is obtained in the WPF step.

\section{Results}

\subsection{Filtration experiments}

Table 4 summarizes the filtration results.

Before filtration, the solution had a cloudy aspect and yellow coloration. After any of the filtrations, the resulting solution had a clear/transparent aspect. This change in the aspect of the solution indicates that undissolved material was retained in the filters.

Analysis of Table 4 reveals that the non-aged solution (filtration 1) resulted in a large FR and a significant viscosity loss even for the relatively large $8 \mu \mathrm{m}$ mesh. This result indicates that this solution would probably cause severe damage in the injection face/well if injected, not being suitable for EOR applications. The same effect can be observed for the $1.2 \mu \mathrm{m}$ mesh filtration of the aged solution (filtration 3).

Differently, the filtrations 2 and 4 presented low FR and viscosity losses, i.e., good filterability. However, only the filtration 4 considered the $1.2 \mu \mathrm{m}$ mesh and, thus, can be considered for core flooding aiming to represent flow deep within the reservoir. One can see that the average viscosity loss experienced by filtration 4 was just 0.1 percentage points above its pre-filtration (equivalent to filtration 2). Additionally, as seen in Table 4 , the filtration 4 has the lowest $\mathrm{FR}_{250}$, indicating it was the best filtration among the tested approaches, albeit still above the limit of 1.2 set by some authors (Gerlach et al., 2019; Sheng, 2011). Therefore, we can say that the $8 \mu \mathrm{m}$ filter was effective in removing almost all the undissolved material (debris from the fabrication process and polymer agglomerates) present in the original solution. This result indicates that the aging plus filtration with the $8 \mu \mathrm{m}$ can be adapted or improved to be used during the biopolymer fabrication/extraction process to improve the quality of the produced polymer powder.

\subsection{Single-phase core flooding}

Table 5 summarizes the RF, RRF, and permeability data obtained through the pressure taps 1,2 , and 3 . Note that the pressure sensor for the pressure drop across the core started malfunctioning during the experiment and did not obtain reliable data.

One can see that the permeability of the sections measured by each pressure tap is similar, as are the RF and RRF measurements. This result indicates data consistency. The RF tended to decrease as the flow rate increased, which is due to the shear-thinning behavior of the scleroglucan solution. Through the RF and RRF data, we could obtain the polymer in-situ viscosity, as shown in Figure 8.

Figure 8 reveals that the in-situ viscosity was slightly lower than the bulk one, consistent with literature for xanthan gum and polyacrylamides under low shear rates (Chauveteau and Zaitoun, 1981; Chauveteau et al., 1984; Stavland et al., 2010). The same behavior was observed for the biopolymer schizophyllan (EOR-grade), which is very similar to scleroglucan (Quadri et al., 2015). Figure 8 also shows that the scleroglucan has a strictly shear thinning behavior, as opposed to HPAM's apparent shear thickening behavior for high shear rates $\left(>300 \mathrm{~s}^{-1}\right.$ ) (Ferreira, 2019; Masalmeh et al., 2019; Stavland et al., 2010). This lack of shear thickening behavior was observed for EORgrade scleroglucan (Jensen et al., 2018) and is an advantage for the scleroglucan, as this behavior can impair injection. Table 6 summarizes the Power-law fit parameters for each curve shown in Figure 8.

Note that the fits for pressure taps 1 and 3 yield similar parameters, while the one for the tap 2 diverges a bit. This difference is mainly due to the tap 2 measurement in $100 \mathrm{~s}^{-1}$, which is considerably higher than the points for taps 1 and $3(2.3 \pm 0.3 \mathrm{mPa} \mathrm{s}$ versus $1.8 \pm 0.1 \mathrm{mPa} \mathrm{s}$ and $1.9 \pm 0.4 \mathrm{mPa}$ s, respectively). That value also causes the $R^{2}$ for the tap 2 to be lower than the other fitted data, which is additional evidence that such value is an outlier.

One crucial result shown by Table 6 is that the behavior index is higher for in-situ viscosity than the bulk one, i.e., the tested polymer solutions are less shear-thinning when flowing through a porous medium than in bulk. The shear-thinning behavior is desirable for EOR applications because the solution displays a lower viscosity near wellbores (i.e., high shear rate), favoring injectivity, while keeping a high viscosity far from wells (i.e., low shear rates), thus improving sweep efficiency.

We can also estimate the in-situ viscosity loss relative to the bulk viscosity, as shown by Figure 9 .

One can see in Figure 9 that the viscosity loss for low shear rates is high (upwards of $24 \% \pm 4 \%$ ), and tends to diminish as shear rate increases. For high shear rates, there are inversions in the viscosity loss behavior, leading to higher in-situ viscosities than the bulk one. This inversion point occurs at around $550 \mathrm{~s}^{-1}$. This inversion occurs because the in-situ viscosity is less shear thinning than the bulk one for this case. 
Table 4. Summary of the filtration experiments results.

\begin{tabular}{|c|c|c|c|c|c|c|}
\hline Filtration & Aging & Pre-filtration & $\begin{array}{c}\text { Filtration } \\
\text { temperature }\left({ }^{\circ} \mathrm{C}\right)\end{array}$ & $\begin{array}{l}\text { Filter mesh } \\
\quad(\mu \mathrm{m})\end{array}$ & $\mathrm{FR}_{250}$ & $\begin{array}{c}\text { Average viscosity } \\
\text { loss }(\%) \\
\end{array}$ \\
\hline 1 & No & No & Ambient $(\sim 25)$ & 8 & 106 & 65.9 \\
\hline 2 & $24 \mathrm{~h}$ at $90^{\circ} \mathrm{C}$ & No & 90 & 8 & 2.91 & 21.2 \\
\hline 3 & $24 \mathrm{~h}$ at $90^{\circ} \mathrm{C}$ & No & 90 & 1.2 & 5.37 & 70.1 \\
\hline 4 & $24 \mathrm{~h}$ at $90^{\circ} \mathrm{C}$ & $8 \mu \mathrm{m}^{2^{*}}$ & 90 & 1.2 & 1.51 & 21.3 \\
\hline
\end{tabular}

* Equivalent to filtration 2.

Table 5. Resistance factor, residual resistance factor, and permeability measured in each pressure tap for each polymer and brine injections.

\begin{tabular}{lcccc}
\hline Injection & \multicolumn{1}{c}{ Property } & Tap 1 & Tap 2 & Tap 3 \\
\hline- & Position relative to injection face & $6.35 \pm 0.01 \mathrm{~cm}$ & $11.43 \pm 0.01 \mathrm{~cm}$ & $16.51 \pm 0.01 \mathrm{~cm}$ \\
First Polymer & $\mathrm{RF}$ at $1 \mathrm{~cm}^{3} / \mathrm{min}$ & $3.71 \pm 0.04$ & $3.69 \pm 0.09$ & $3.77 \pm 0.19$ \\
Second Polymer & $\mathrm{RF}$ at $0.25 \mathrm{~cm}^{3} / \mathrm{min}$ & $6.02 \pm 0.29$ & $8.18 \pm 0.96$ & $8.04 \pm 1.64$ \\
Second Polymer & $\mathrm{RF}$ at $0.5 \mathrm{~cm}^{3} / \mathrm{min}$ & $5.27 \pm 0.13$ & $5.52 \pm 0.27$ & $6.46 \pm 0.68$ \\
Second Polymer & $\mathrm{RF} \mathrm{at} 1 \mathrm{~cm}^{3} / \mathrm{min}$ & $4.31 \pm 0.05$ & $4.53 \pm 0.11$ & $5.58 \pm 0.28$ \\
Second Polymer & $\mathrm{RF}$ at $1.5 \mathrm{~cm}^{3} / \mathrm{min}$ & $4.19 \pm 0.03$ & $4.41 \pm 0.07$ & $5.42 \pm 0.19$ \\
Second Polymer & $\mathrm{RF}$ at $2 \mathrm{~cm}^{3} / \mathrm{min}$ & $4.09 \pm 0.03$ & $4.28 \pm 0.05$ & $5.14 \pm 0.13$ \\
First Brine & Permeability to brine & $373 \pm 3 \mathrm{mD}$ & $489 \pm 9 \mathrm{mD}$ & $407 \pm 12 \mathrm{mD}$ \\
Second Brine & $\mathrm{RRF}$ at $1 \mathrm{~cm}^{3} / \mathrm{min}$ & $1.79 \pm 0.02$ & $1.93 \pm 0.05$ & $2.37 \pm 0.12$ \\
Third Brine & $\mathrm{RRF}$ at $1 \mathrm{~cm}^{3} / \mathrm{min}$ & $1.88 \pm 0.02$ & $1.93 \pm 0.05$ & $2.36 \pm 0.12$ \\
\hline
\end{tabular}

${ }^{*}$ Value obtained through the average of measurements done at $0.25,0.5,1,1.5$ and $2 \mathrm{~cm}^{3} / \mathrm{min}$.

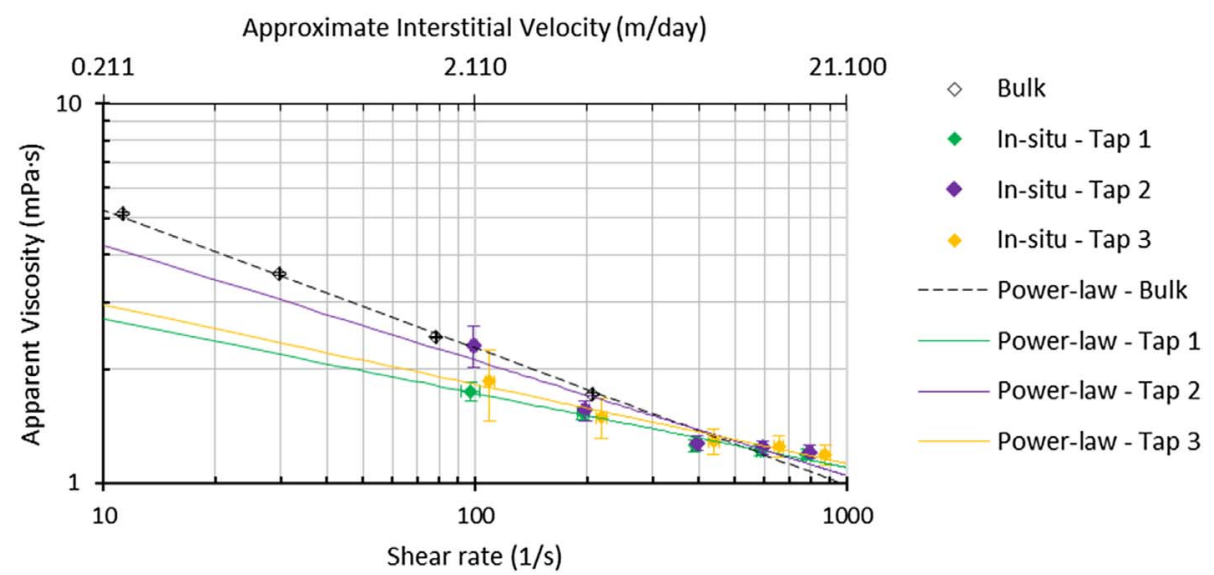

Fig. 8. Bulk and in-situ viscosity of 500 ppm scleroglucan in SSW.

It is worth noting that the designed polymer solution lost on average $21.3 \%$ of its viscosity after the filtration process, and the in-situ viscosity presented an additional loss when compared with the bulk viscosity during the single-phase core flooding test. That shows that forecasts involving polymer flow in porous media performed with bulk and/or unfiltered data may be inaccurate.

The adsorption of scleroglucan in the system studied in this work was measured as $20.8 \pm 1.4 \mu \mathrm{g} / \mathrm{g}$. This retention level is considered low and is consistent with some literature (Fournier et al., 2018; Rivenq et al., 1992). Rivenq et al. (1992) found $30 \mu \mathrm{g} / \mathrm{g}$ retention of $400 \mathrm{ppm}$ non-EOR-grade scleroglucan in Berea sandstone at $90{ }^{\circ} \mathrm{C}$. Fournier et al. (2018) measured the retention of an EOR-grade scleroglucan at $50{ }^{\circ} \mathrm{C}$ in Berea sandstone $(1470 \mathrm{mD})$ and Estaillades limestone $(120 \mathrm{mD})$ and found $12 \mu \mathrm{g} / \mathrm{g}$ for the first formation, and $8 \mu \mathrm{g} / \mathrm{g}$ for the second. On the other hand, Kulawardana et al. (2012) observed $211 \mu \mathrm{g} / \mathrm{g}$ of 
Table 6. Power-law fits of the rheology data shown in Figure 8.

\begin{tabular}{lccc}
\hline $\begin{array}{l}\text { Power-law } \\
\text { Fit } \eta=K \cdot \dot{\gamma}^{n-1}\end{array}$ & Consistency index $(K)$ & Behavior index $(n)$ & $\begin{array}{c}\text { Coefficient of } \\
\text { determination }\left(R^{2}\right)\end{array}$ \\
\hline Bulk & 12.1 & 0.638 & 0.989 \\
In-situ - Tap 1 & 4.26 & 0.804 & 0.965 \\
In-situ - Tap 2 & 8.55 & 0.696 & 0.900 \\
In-situ - Tap 3 & 4.77 & 0.791 & 0.960 \\
\hline
\end{tabular}

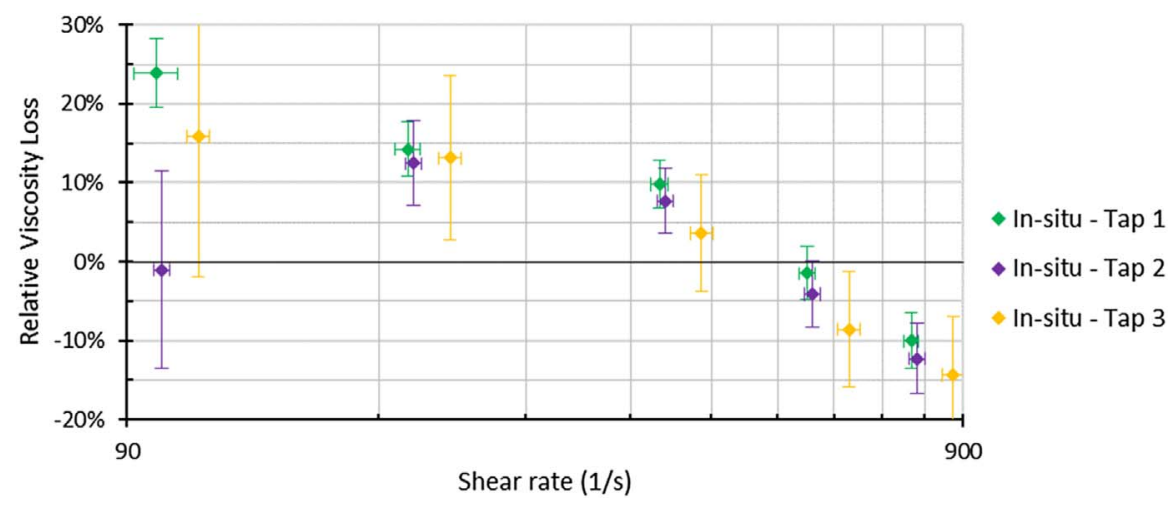

Fig. 9. Viscosity loss relative to bulk rheology versus shear rate for the in-situ viscosity measurements in each pressure tap.

1250 ppm EOR-grade scleroglucan in $278 \mathrm{mD}$ Berea sandstone at $50{ }^{\circ} \mathrm{C}$. Liang et al. (2019) found $127 \mu \mathrm{g} / \mathrm{g}$ of $2500 \mathrm{ppm}$ scleroglucan in $303 \mathrm{mD}$ artificial sand cores at $85^{\circ} \mathrm{C}$. The low adsorption in carbonates observed in this work is one advantage of the nonionic scleroglucan over anionic polyacrylamides, which can present very high adsorption in the presence of calcium carbonate due to surface charges (Saboorian-Jooybari et al., 2016). For instance, Masalmeh et al. (2019) found retention values of upwards of $570 \mu \mathrm{g} / \mathrm{g}$ for an anionic polyacrylamide in carbonate.

In this study, we measured an IAPV of $4.4 \% \pm 0.1 \%$, which is also considered low. This low IAPV is consistent with the result that showed that in-situ viscosity is only slightly lower than bulk. According to literature, the IAPV has an indirect effect in the in-situ viscosity through the depleted layer effect, and the higher the IAPV, the higher is the reduction in in-situ viscosity relative to bulk one (Stavland et al., 2010).

\subsection{Two-phase core flooding}

Table 7 summarizes the production properties of both oil recovery processes (waterflooding and polymer flooding), along with the respective oil injection steps preceding the recovery (O1 and $\mathrm{O} 2)$.

One can see in Table 7 that even though the irreducible water saturations $\left(s_{\mathrm{wi}}\right)$ of both oil injections were similar, the oil effective permeabilities $\left(k_{\mathrm{o}}\right)$ at such conditions were very different. This behavior indicates that a permeability hysteresis phenomenon is in place, which is known to happen in resaturation processes (Braun and Holland, 1995). We can attribute this permeability hysteresis to phase trapping or contact angle hysteresis (Dernaika et al., 2012; Masalmeh, 2002).
The polymer flooding was able to delay the water phase breakthrough when compared to waterflooding (0.117 versus 0.043 injected $\mathrm{PV}$ ), indicating that the oil displacement was more stable under polymer flooding. Davison and Mentzer (1982) used 625 ppm non-EOR-grade scleroglucan (seawater, room temperature) in $1 / 4$ of five-spot ballotini packs with field oil from North Sea reservoirs and observed breakthrough delays between 0.4 and 0.11 injected PV when compared to waterflooding. These breakthrough delays are comparable to core floodings in Indiana limestone (between 80 and $90 \mathrm{mD}$ ) using brine (synthetic sea water of $5.7 \%$ ) and an HPAM polymer solution (Flopaam 3230S, $2500 \mathrm{ppm}$ in the same synthetic sea water), which reported a breakthrough of 0.142 for brine and 0.305 for polymer (Gao, 2014). However, the experiments reported by Gao (2014) required a much higher polymer concentration than the one used in this work as scleroglucan is more resistant to salinity than HPAM.

Additionally, the PF managed to anticipate the $95 \%$ water cut instant relative to WF (4.74 versus 6.66 injected $\mathrm{PV}$ ), which is a consequence of the oil recovery anticipation characteristic of the PF process. This oil recovery anticipation can also be seen by the significant oil recovery increase resulting from the PF, as Figure 10 shows along with the water-oil ratios.

As Table 7 and Figure 10 show, oil recovery by scleroglucan solution resulted in oil recovery anticipation and increased ultimate oil recovery. The increment on final oil recovery was $6.3 \% \pm 6.8 \%$. Jensen et al. (2018) observed a $15 \%$ increase in ultimate oil recovery by applying tertiary polymer flooding (2000 ppm of EOR-grade SCLG) after the oil recovery by waterflooding had reached a plateau in a $726 \mathrm{mD}$ Berea sandstone. Similarly, Gao (2014) reported a $7.2 \%$ incremental oil recovery for tertiary flooding of 
Table 7. Relevant production properties of both oil injections (O1 and $\mathrm{O} 2$ ) and both oil recovery methods (WF and $\mathrm{PF})$.

\begin{tabular}{|c|c|c|c|c|}
\hline Property & & Unit & $\begin{array}{l}\text { First oil injection } \\
(\mathrm{O} 1)\end{array}$ & $\begin{array}{c}\text { Second oil injection } \\
(\mathrm{O} 2)\end{array}$ \\
\hline Start of Test & Irreducible Water Saturation & - & $50.9 \% \pm 2.7 \%$ & $50.4 \% \pm 4.3 \%$ \\
\hline Start of Test & $\begin{array}{l}\text { Oil Effective Permeability at Irreducible Water } \\
\text { Saturation }\end{array}$ & $\mathrm{mD}$ & $266.1 \pm 7.6$ & $152.9 \pm 4.5$ \\
\hline Start of Test & Oil Mobility & $\mathrm{mD} / \mathrm{cP}$ & $40.0 \pm 1.3$ & $23.0 \pm 0.7$ \\
\hline Property & & Unit & $\begin{array}{l}\text { Waterflooding } \\
\text { (WF) }\end{array}$ & $\begin{array}{l}\text { Polymer flooding } \\
\text { (PF) }\end{array}$ \\
\hline Breakthrough & Injected Pore Volume & - & $0.043 \pm 0.007$ & $0.117 \pm 0.008$ \\
\hline Breakthrough & Cumulative Produced Oil & $\mathrm{cm}^{3}$ & $1.85 \pm 0.21$ & $4.85 \pm 0.21$ \\
\hline Breakthrough & Oil Recovery Factor & - & $6.9 \% \pm 0.9 \%$ & $17.9 \% \pm 1.9 \%$ \\
\hline $95 \%$ Water Cut & Injected Pore Volume & - & $6.66 \pm 0.16$ & $4.74 \pm 0.11$ \\
\hline $95 \%$ Water Cut & Cumulative Produced Oil & $\mathrm{cm}^{3}$ & $11.6 \pm 0.2$ & $12.9 \pm 0.2$ \\
\hline $95 \%$ Water Cut & Oil Recovery Factor & - & $43.2 \% \pm 3.1 \%$ & $47.7 \% \pm 4.6 \%$ \\
\hline $95 \%$ Water Cut & Cumulative Water-Oil Ratio & - & $30.4 \pm 0.6$ & $19.0 \pm 0.3$ \\
\hline End of Test & Injected Pore Volume & - & $25.0 \pm 0.6$ & $27.7 \pm 0.7$ \\
\hline End of Test & Cumulative Produced Oil & $\mathrm{cm}^{3}$ & $14.2 \pm 0.2$ & $16.2 \pm 0.2$ \\
\hline End of Test & Oil Recovery Factor & - & $52.9 \% \pm 3.7 \%$ & $59.7 \% \pm 5.8 \%$ \\
\hline End of Test & Cumulative Water-Oil Ratio & - & $94.2 \pm 1.4$ & $91.5 \pm 1.2$ \\
\hline End of Test & Residual Oil Saturation & - & $23.1 \% \pm 3.4 \%$ & $20.0 \% \pm 4.8 \%$ \\
\hline End of Test & $\begin{array}{l}\text { Water Effective Permeability at Residual Oil } \\
\text { Saturation }\end{array}$ & $\mathrm{mD}$ & $52.7 \pm 2.8^{*}$ & $67.6 \pm 3.5^{*}$ \\
\hline End of Test & Resistance Factor & - & - & $2.28 \pm 0.02^{*}$ \\
\hline End of Test & Residual Resistance Factor & - & - & $0.78 \pm 0.01^{*}, \dagger$ \\
\hline End of Test & In-situ Viscosity & $\mathrm{mPas}$ & $0.545 \pm 0.016$ & $1.60 \pm 0.03^{*}$ \\
\hline End of Test & Water Terminal Mobility & $\begin{array}{l}\mathrm{mD} / \\
\mathrm{mPa} \mathrm{s}\end{array}$ & $119 \pm 3^{*}$ & $42.4 \pm 2.3^{*}$ \\
\hline End of Test & Terminal Mobility Ratio & - & $2.98 \pm 0.12$ & 1.840 .12 \\
\hline
\end{tabular}

\footnotetext{
* Data relative to the second pressure tap.

${ }^{\dagger}$ Datum obtained in the water post-flooding step.
}

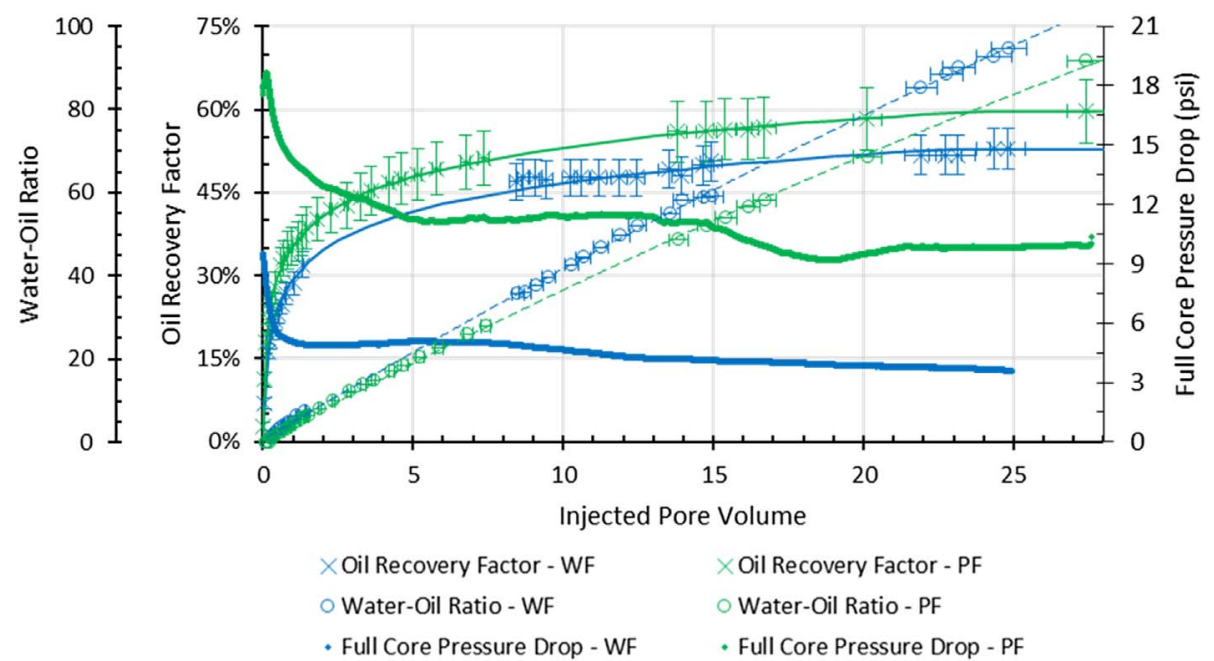

Fig. 10. Oil recovery factor (exes), water-oil rato (circles) and full core pressure drop (diamonds) histories for the waterflooding (WF, blue) and polymer flooding ( $\mathrm{PF}$, green) injections. The lines are meant only to guide the eyes and were not used to determine properties. 

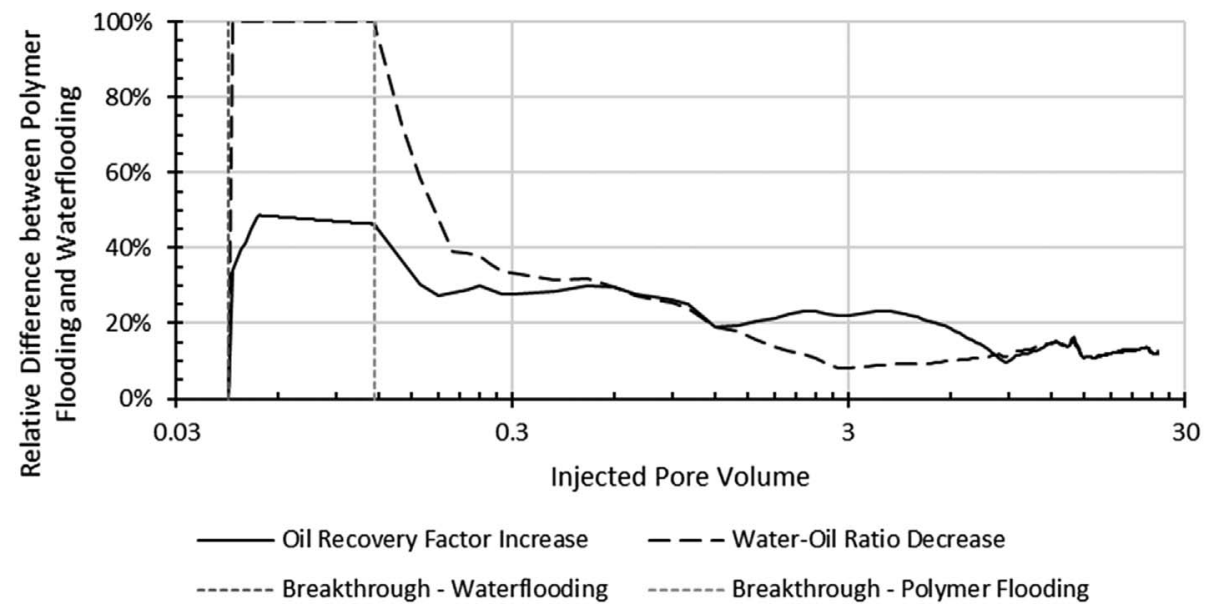

Fig. 11. Relative differences of oil recovery increase (full line) and water-oil ratio decrease (dotted line) for the polymer flooding relative to the waterflooding.

Table 8. Permeabilities, resistance factors, and polymer in-situ viscosities measured by each pressure sensor and in each injection.

\begin{tabular}{|c|c|c|c|c|c|}
\hline \multirow[t]{2}{*}{ Property } & \multirow[t]{2}{*}{ Unit } & \multicolumn{4}{|c|}{ Measurement point } \\
\hline & & Full core & Pressure tap 1 & Pressure tap 2 & Pressure tap 3 \\
\hline Position relative to injection face & $\mathrm{cm}$ & 0 & $11.43 \pm 0.01$ & $16.51 \pm 0.01$ & $21.59 \pm 0.01$ \\
\hline Absolute permeability & $\mathrm{mD}$ & $336 \pm 4$ & $334 \pm 5$ & $301 \pm 6$ & $264 \pm 7$ \\
\hline$k_{\mathrm{o}}$ at $s_{\mathrm{wi}}$ for $\mathrm{O} 1$ & $\mathrm{mD}$ & $266 \pm 8$ & $235 \pm 10$ & $217 \pm 12$ & $197 \pm 14$ \\
\hline$k_{\mathrm{w}}$ at $s_{\mathrm{or}}$ for $\mathrm{WF}$ & $\mathrm{mD}$ & $65.0 \pm 1.6$ & $66.3 \pm 2.9$ & $52.7 \pm 2.8$ & $42.1 \pm 3.0$ \\
\hline$k_{\mathrm{o}}$ at $s_{\mathrm{wi}}$ for $\mathrm{O} 2$ & $\mathrm{mD}$ & $155 \pm 5$ & $182 \pm 9$ & $170 \pm 9$ & $158 \pm 11$ \\
\hline$k_{\mathrm{w}}$ at $s_{\mathrm{or}}$ for WPF & $\mathrm{mD}$ & $-^{*}$ & $76.9 \pm 3.2$ & $67.6 \pm 3.5$ & $57.0 \pm 4.0$ \\
\hline $\mathrm{RF}$ at $s_{\mathrm{or}}$ for $\mathrm{PF}$ & - & $2.73 \pm 0.01$ & $2.54 \pm 0.04$ & $2.28 \pm 0.02$ & $2.16 \pm 0.02$ \\
\hline RRF at $s_{\text {or }}$ for WPF & - & $-{ }^{*}$ & $0.86 \pm 0.01$ & $0.78 \pm 0.01$ & $0.74 \pm 0.01$ \\
\hline$\eta_{\mathrm{p}, \text { in-situ }}$ at $s_{\mathrm{or}}$ for $\mathrm{PF}$ & $\mathrm{mPa} \mathrm{s}$ & $-^{*}$ & $1.61 \pm 0.04$ & $1.60 \pm 0.03$ & $1.59 \pm 0.02$ \\
\hline$\dot{\gamma}_{\text {is }}$ & $\mathrm{s}^{-1}$ & $-^{*}$ & $357 \pm 15$ & $381 \pm 17$ & $415 \pm 21$ \\
\hline
\end{tabular}

* These properties could not be estimated due to a pressure sensor malfunction.

HPAM (Flopaam 3230S, 2500 ppm in 5.7\% TDS SSW) in Indiana limestone. Literature generally attributes the reduction of residual oil saturation by polymer flooding to the solution viscoelasticity (Boekhout, 2015; Wang et al., 2000, 2001, 2007). Liang et al. (2019) reported that scleroglucan exhibits a viscoelastic behavior under high salinity $(101,000 \mathrm{ppm}$ TDS $)$ and temperature $\left(85^{\circ} \mathrm{C}\right)$ conditions, even though the lowest polymer concentration tested by those authors was $1000 \mathrm{ppm}$. Nevertheless, Wang et al. (2007) observed an incremental oil after $s_{\text {or }}$ was reached even for non-viscoelastic xanthan gum (between $2 \%$ and $8 \%$ ), albeit this additional oil was lower than that obtained for viscoelastic polyacrylamide (between $5 \%$ and $23 \%$ ).

Additionally, one can see in Table 7 and Figure 10 that the cumulative WOR were considerably lower for PF relative to WF. This result represents another advantage of the polymer flooding, which is the ability to reduce the produced water volumes.
Figure 11 depicts the relative differences between polymer flooding and waterflooding in terms of oil recovery factor increase and water-oil ratio decrease.

Figure 11 shows that the most significant advantages of the polymer flooding process are observed for low injected pore volumes $(<1 \mathrm{Inj}$. PV), primarily until the polymer breakthrough. Both the ORF relative increase and the WOR relative decrease remain high until the polymer breakthrough and then start decreasing from this point on.

It is important to highlight that we could achieve these benefits for polymer flooding under a low polymer concentration (500 ppm) and marginal viscosity and mobility ratio improvements. Solution viscosity increased from $0.545 \pm 0.016 \mathrm{mPa} \mathrm{s}$ in the WF to $1.60 \pm 0.03 \mathrm{mPa} \mathrm{s}$ in the $\mathrm{PF}$, while terminal fluid mobility respectively decreased from $119 \pm 3 \mathrm{mD} / \mathrm{mPa}$ s to $42.4 \pm 2.3 \mathrm{mD} / \mathrm{mPa}$ s. Besides the three-fold increase in viscosity and $64 \%$ reduction in 
terminal mobility, we only observed a $38 \% \pm 3 \%$ reduction in terminal mobility ratio from $2.98 \pm 0.12 \mathrm{mD} / \mathrm{mPa} \mathrm{s}$ (WF/O1) to $1.84 \pm 0.12 \mathrm{mD} / \mathrm{mPa}$ s $(\mathrm{PF} / \mathrm{O} 2)$. This lessthan-expected mobility reduction is due to different oil terminal effective permeabilities for each of these steps (O1 for WF and O2 for PF, see Tab. 7).

Our experiments show evidence that oil recovery can be enhanced even for small mobility ratio reductions, and this can be achieved for low concentration polymer flooding. It is worth noting that this work was developed with core flooding experiments and, thus, is representative of the displacement efficiencies. Additional studies are necessary to evaluate the areal and vertical sweep efficiencies and further investigate if low concentration polymer flooding is advantageous.

Table 8 summarizes the data derived from the pressure measurements along with the in-situ shear rate. Note that the full core pressure sensor malfunctioned during the water post flooding, making some properties unable to estimate.

Table 8 shows that the permeability measured tends to reduce further from the injection face, indicating a lower permeability zone near the production face and a longitudinal heterogeneity.

The in-situ viscosity data indicates that the polymer viscosity measured in the two-phase core flooding experiment was slightly higher than the one estimated through the singlephase core flooding. For example, in the single-phase experiment, the viscosity measured at $438 \pm 14 \mathrm{~s}^{-1}$ was $1.29 \pm 0.10 \mathrm{mPa}$ s, while the $415 \pm 21 \mathrm{~s}^{-1}$ viscosity measured in the two-phase core flooding was $1.59 \pm$ $0.02 \mathrm{mPa}$ s. This result indicates that the presence of an oil phase may lead to distinct pore-scale transport characteristics for polymer, resulting in different observations for macroscopic viscosity measurements.

\section{Conclusion}

This paper conducted an experimental investigation of low concentration scleroglucan solutions with a focus on EOR in offshore carbonate reservoirs. The main findings are:

- The tested solution presented good filterability in a two-stage Millipore filtration $(8 \mu \mathrm{m}$ then $1.2 \mu \mathrm{m})$ at $90{ }^{\circ} \mathrm{C}$ after a $24 \mathrm{~h}$ aging process. Filtration in $8 \mu \mathrm{m}$ without the aging period was poor, indicating that this non-EOR-grade scleroglucan without pre-treatment solution may have poor performance near-wellbore.

- The 500 ppm scleroglucan solution showed low retention $(20.8 \mu \mathrm{g} / \mathrm{g})$, consistent with other literature on scleroglucan solutions and indicating that the filtration process was effective.

- The measured permeability reduction was low, consistent with the low retention observation.

- The in-situ viscosity was slightly smaller than the bulk one, with measurements in the two-phase core flooding indicating marginally higher values than those obtained in a correspondent single-phase experiment. This result suggests that the tested scleroglucan solution maintains its viscosity capabilities while flowing through porous media. However, the in-situ viscosity was less shear-thinning than the bulk one, dampening the advantage of low viscosity in high shear zones (e.g., wells).

- The measured inaccessible pore volume was low $(4.4 \%)$, consistent with the observation of small viscosity reduction between in-situ and bulk.

- We observed several oil displacement advantages of the tested scleroglucan solution over conventional waterflooding even with marginal improvements on viscosity $(0.545-1.60 \mathrm{mPa} \mathrm{s})$ and terminal mobility ratio (2.98-1.84). The tested polymer solution was able to delay the water phase breakthrough, anticipate oil recovery, increase ultimate oil recovery, and considerably reduce water-oil ratio.

- Our experiments show evidence that oil recovery can be enhanced even for small mobility ratio reductions during polymer flooding. However, our tests are representative of the displacement efficiency and, thus, additional evaluations are necessary to include volumetric sweep efficiencies and further investigate the global advantages for low concentration polymer flooding.

Acknowledgments. This research was carried out in association with the ongoing R\&D project registered as ANP 20359-6, "Injeção de Biopolímeros para a Recuperação Avançada de Petróleo de Reservatórios do Pré-Sal Brasileiro" (University of Campinas/Shell Brasil/ANP) - Biopolymer Injection for Enhanced Oil Recovery in Brazilian Pre-Salt Reservoirs, sponsored by Shell Brasil under the ANP R\&D levy as "Compromisso de Investimentos com Pesquisa e Desenvolvimento". This project is carried out in partnership with Petrobras.

\section{References}

Al-Adasani A., Bai B. (2010) Recent developments and updated screening criteria of enhanced oil recovery techniques, in: International Oil and Gas Conference and Exhibition in China, Society of Petroleum Engineers, pp. 1-24. doi: 10.2118/130726-MS.

Al-Hadhrami H.S., Blunt M.J. (2000) Thermally induced wettability alteration to improve oil recovery in fractured reservoirs, in: SPE/DOE Improved Oil Recovery Symposium, Society of Petroleum Engineers, pp. 179-186. doi: 10.2118/ 59289-MS.

API. (1998) Recommended Practices for Core Analysis, Author. API. (1990) Recommended Practices for Evaluation of Polymers Used in Enhanced Oil Recovery Operations, Author.

Baijal S.K., Dey N.C. (1982) Role of molecular parameters during flow of polymer solutions in unconsolidated porous media, J. Appl. Polym. Sci. 27, 121-131. doi: 10.1002/ app.1982.070270114.

Beall B., Monroe T., Vollmer D., Hanna F. (2004) Brine effects on hydration rates for polymers used in completion and workover operations, in: Proceedings of SPE International Symposium and Exhibition on Formation Damage Control, Society of Petroleum Engineers, pp. 431-444. doi: 10.2523/86505-MS. 
Boekhout S.G. (2015) Developing a workflow for a study of polymer flooding in heterogeneous reservoirs, Delft University of Technology.

Bourdarot G., Ghedan S.G. (2011) Modified EOR screening criteria as applied to a group of offshore carbonate oil reservoirs, in: SPE Reservoir Characterisation and Simulation Conference and Exhibition, Society of Petroleum Engineers, pp. 1-21. doi: 10.2118/148323-MS.

Braun E.M., Holland R.F. (1995) Relative permeability hysteresis: Laboratory measurements and a conceptual model, SPE Reserv. Eng. 10, 222-228. doi: 10.2118/28615-PA.

Cannella W.J., Huh C., Seright R.S. (1988) Prediction of xanthan rheology in porous media, in: SPE Annual Technical Conference and Exhibition, Society of Petroleum Engineers. doi: 10.2118/18089-MS.

Carcoana A.N. (1982) Enhnced oil recovery in Rumania, in: SPE Enhanced Oil Recovery Symposium, Society of Petroleum Engineers. doi: 10.2118/10699-MS.

Chang H.L. (1978) Polymer Flooding Technology Yesterday, Today, and Tomorrow, J. Pet. Technol. 30, 1113-1128. doi: 10.2118/7043-PA.

Chauveteau G., Lecourtier J. (1988) Propagation of polymer slugs through adsorbent porous media, Water-Soluble Polymers for Petroleum Recovery, Springer, US, Boston, MA, pp. 53-68. doi: 10.1007/978-1-4757-1985-7_3.

Chauveteau G., Tirrell M., Omari A. (1984) Concentration dependence of the effective viscosity of polymer solutions in small pores with repulsive or attractive walls, J. Colloid Interface Sci. 100, 41-54. doi: 10.1016/0021-9797(84)90410-7.

Chauveteau G., Zaitoun A. (1981) Basic rheological behavior of xanthan polysaccharide solutions in porous media: effects of pore size and polymer concentration, in: Proceedings to the European Symposium on EOR, pp. 197-212.

Davison P., Mentzer E. (1982) Polymer flooding in North Sea reservoirs, Soc. Pet. Eng. J. 22, 353-362. doi: 10.2118/ 9300-PA.

Dawson R., Lantz R.B. (1972) Inaccessible pore volume in polymer flooding, Soc. Pet. Eng. J. 12, 448-452. doi: 10.2118/ 3522-PA.

de Melo M.A., da Silva I.P.G., de Godoy G.M.R., Sanmartim A.N. (2002) Polymer injection projects in brazil: dimensioning, field application and evaluation, in: $S P E / D O E$ Improved Oil Recovery Symposium, Society of Petroleum Engineers, pp. 11. doi: 10.2118/75194-MS.

Delamaide E., Bazin B., Rousseau D., Degre G. (2014) Chemical EOR for heavy oil: The Canadian experience, in: SPE EOR Conference at Oil and Gas West Asia, Society of Petroleum Engineers. doi: 10.2118/169715-MS.

Demin W., Zhang Z., Chun L., Cheng J., Du X., Li Q. (1996) A pilot for polymer flooding of Saertu formation S II 10-16 in the North of Daqing oil field, SPE Asia Pacific Oil and Gas Conference, Society of Petroleum Engineers, pp. 431-441. doi: 10.2118/37009-MS.

Dernaika M.R., Kalam M.Z., Basioni M.A., Skjæveland S.M. (2012) Hysteresis of capillary pressure, resistivity index and relative permeability in different carbonate rock types, Petrophysics 53, 316-332.

Dupuis G., Rousseau D., Tabary R., Grassl B. (2011) Flow of hydrophobically modified water-soluble-polymer solutions in porous media: New experimental insights in the diluted regime, SPE J. 16, 43-54. doi: 10.2118/129884-PA.

El-Haddad M.N. (2014) Hydroxyethylcellulose used as an ecofriendly inhibitor for 1018 c-steel corrosion in $3.5 \% \mathrm{NaCl}$ solution, Carbohydr. Polym. 112, 595-602. doi: 10.1016/ j.carbpol.2014.06.032.

Fariña J.I., Siñeriz F., Molina O.E., Perotti N.I. (2001) Isolation and physicochemical characterization of soluble scleroglucan from Sclerotium rolfsii. Rheological properties, molecular weight and conformational characteristics, Carbohydr. Polym. 44, 41-50. doi: 10.1016/S0144-8617(00)00189-2.

Ferreira V.H.S. (2019) Key parameters on the flow of polymer solutions through porous medium - Experimental and modeling studies, University of Campinas.

Fournier R., Tiehi J., Zaitoun A. (2018) Laboratory study of a new EOR-Grade Scleroglucan, in: SPE EOR Conference at Oil and Gas West Asia, Society of Petroleum Engineers, pp. 26-28. doi: 10.2118/190451-MS.

Gallino G., Guarneri A., Poli G., Xiao L. (1996) Scleroglucan biopolymer enhances WBM performances, in: SPE Annual Technical Conference and Exhibition, Society of Petroleum Engineers'. doi: 10.2118/36426-MS.

Gao C. (2016) Potential applications of Welan gum in upstream petroleum industry, Int. J. Oil Gas Coal Eng. 4, 16. doi: 10.11648/j.ogce.20160402.12.

Gao J. (2014) An experimental polymer EOR study of a high salinity low permeability carbonate reservoir, University of Calgary. doi: 10.11575/PRISM/24888.

Gerlach B., Dugonjic-Bilic F., Neuber M., Alkouh A. (2019) Best surfactant for EOR polymer injectivity, in: Soc. Pet. Eng. SPE Kuwait Oil Gas Show Conf. 2019, KOGS 2019. doi: 10.2118/198097-MS.

Ghannam M.T., Esmail M.N. (1998) Rheological properties of aqueous polyacrylamide solutions, J. Appl. Polym. Sci. 69, 1587-1597. doi: 10.1002/(SICI)1097-4628(19980822)69:8< 1587::AID-APP13>3.0.CO;2-S.

Goddard E.D., Gruber J.V. (1999) Principles of polymer science and technology in cosmetics and personal care, 1st edn., CRC Press.

He L., Zhao L., Li J., Ma J., Lui R., Wang S., Zhao W. (2014) Complex relationship between porosity and permeability of carbonate reservoirs and its controlling factors: A case study of platform facies in Pre-Caspian Basin, Pet. Explor. Dev. 41, 225-234. doi: 10.1016/S1876-3804(14)60026-4.

Heemskerk J., Janssen-van Rosmalen R., Holtslag R.J., Teeuw D. (1984) Quantification of viscoeleastic effects of polyacrylamide solutions, in: SPE/DOE Fourth Symp. Enhanc. Oil Recover., pp. 1-8. doi:10.2118/12652-MS.

Hirasaki G., Zhang D.L. (2004) Surface chemistry of oil recovery from fractured, oil-wet, carbonate formations, SPE J. 9, 151-162. doi: 10.2118/88365-PA.

Huh C., Lange E.A., Cannella W.J. (1990) Polymer retention in porous media, SPE/DOE Enhanced Oil Recovery Symposium, Society of Petroleum Engineers. doi: 10.2118/20235-MS.

Jennings R.R., Rogers J.H., West T.J. (1971) Factors influencing mobility control by polymer solutions, J. Pet. Technol. 23, 391-401. doi: 10.2118/2867-PA.

Jensen T., Kadhum M., Kozlowicz B., Sumner E.S., Malsam J., Muhammed F., Ravikiran R. (2018) Chemical EOR under harsh conditions: Scleroglucan as a viable commercial solution, in: SPE Improved Oil Recovery Conference, Society of Petroleum Engineers, pp. 1-16. doi: 10.2118/190216-MS.

Kaijanen L., Paakkunainen M., Pietarinen S., Jernström E., Reinikainen S.P. (2015) Ultraviolet detection of monosaccharides: Multiple wavelength strategy to evaluate results after capillary zone electrophoretic separation, Int. J. Electrochem. Sci. 10, 2950-2961. 
Kamal M.S., Sultan A.S., Al-Mubaiyedh U.A., Hussein I.A. (2015) Review on polymer flooding: rheology, adsorption, stability, and field applications of various polymer systems, Polym. Rev. 55, 491-530. doi: 10.1080/15583724.2014.982821.

Kang P.-S., Lim J.-S., Huh C. (2016) Screening criteria and considerations of offshore enhanced oil recovery, Energies $\mathbf{9}$, 44. doi: 10.3390/en9010044.

Kang X., Zhang J., Sun F., Zhang F., Feng G., Yang J., Zhang X., Xiang W. (2011) A review of polymer EOR on offshore heavy oil field in Bohai Bay, China, in: SPE Enhanced Oil Recovery Conference, Society of Petroleum Engineers. doi: 10.2118/144932-MS.

Kulawardana E.U., Koh H., Kim D.H., Liyanage P.J., Upamali K., Huh C., Weerasooriya U., Pope G.A. (2012) Rheology and transport of improved EOR polymers under harsh reservoir conditions, in: SPE Improved Oil Recovery Symposium, Society of Petroleum Engineers, pp. 1-14. doi: 10.2118/154294-MS.

Liang K., Han P., Chen Q., Su X., Feng Y. (2019) Comparative study on enhancing oil recovery under high temperature and high salinity: Polysaccharides versus synthetic polymer, ACS Omega 4, 10620-10628. doi: 10.1021/acsomega. $9 \mathrm{~b} 00717$.

Littmann W. (1988) Polymer Flooding, Elsevier Science.

Liu B., Sun X.S., Wang K., Xu H., Liu Q., Liu X., Song S. (2007) Flooded by high concentration polymer doubled oil recovery of common polymer on field test with $20 \%$ closed to the result of lab test in Daqingin: International Oil Conference and Exhibition in Mexico, Society of Petroleum Engineers, pp. 1-9. doi: 10.2118/108684-MS.

Lotsch T., Muller T., Pusch G. (1985) The effect of inaccessible pore volume on polymer coreflood experiments, in: SPE Oilfield and Geothermal Chemistry Symposium, Society of Petroleum Engineers. doi: 10.2118/13590-MS.

Masalmeh S., AlSumaiti A., Gaillard N., Daguerre F., Skauge T., Skuage A. (2019) Extending polymer flooding towards high-temperature and high-salinity carbonate reservoirs, in: Abu Dhabi International Petroleum Exhibition $\&$ Conference, Society of Petroleum Engineers, pp. 1-23. doi: 10.2118/ 197647-MS.

Masalmeh S.K. (2002) The effect of wettability on saturation functions and impact on carbonate reservoirs in the middle east, in: Abu Dhabi Int. Pet. Exhib. Conf., pp. 1-9. doi: 10.2118/78515-MS.

Methemitis C., Morcellet M., Sabbadin J., Francois J. (1986) Interactions between partially hydrolyzed polyacrylamide and ionic surfactants. Eur. Polym. J. 22, 619-627. doi: 10.1016/ 0014-3057(86)90158-8.

Meyer R.F., Attanasi E.D., Freeman P.A. (2007) Heavy oil and natural bitumen resources in geological basins of the world, U.S. Geological Survey.

Moradi-Araghi A., Doe P.H. (1987) Hydrolysis and precipitation of polyacrylamides in hard brines at elevated temperatures, SPE Reserv. Eng. 2, 189-198. doi: 10.2118/13033-PA.

Padsalgikar A.D. (2017) Introduction to plastics, in: Plastics in Medical Devices for Cardiovascular Applications, Elsevier, pp. 1-29. doi: 10.1016/B978-0-323-35885-9.00001-1.

Pereira da Costa M., Conte-Junior C.A. (2015) Chromatographic methods for the determination of carbohydrates and organic acids in foods of animal origin, Compr. Rev. Food Sci. Food Saf. 14, 586-600. doi: 10.1111/1541-4337.12148.

Pu W., Shen C., Wei B., Yang Y., Li Y. (2017) A comprehensive review of polysaccharide biopolymers for enhanced oil recovery (EOR) from flask to field, J. Ind. Eng. Chem. 61 , 1-11. doi: 10.1016/j.jiec.2017.12.034.

Quadri S.M.R., Shoaib M., AlSumaiti A.M., Alhassan S.M. (2015) Screening of polymers for eor in high temperature, high salinity and carbonate reservoir conditions, in: International Petroleum Technology Conference, 6-9 December, Doha, Qatar. doi: 10.2523/IPTC-18436-MS.

Rivenq R.C., Donche A., Nolk C. (1992) Improved scleroglucan for polymer flooding under harsh reservoir conditions, SPE Reserv. Eng. 7, 15-20. doi: 10.2118/19635-PA.

Roy D., Semsarilar M., Guthrie J.T., Perrier S. (2009) Cellulose modification by polymer grafting: a review, Chem. Soc. Rev. 38, 2046. doi: 10.1039/b808639g.

Ruthven D.M. (1984) Principles of adsorption and adsorption processes, Wiley.

Saboorian-Jooybari H., Dejam M., Chen Z. (2016) Heavy oil polymer flooding from laboratory core floods to pilot tests and field applications: Half-century studies, J. Pet. Sci. Eng. 142, 85-100. doi: 10.1016/j.petrol.2016.01.023.

Seright R.S. (2017) How much polymer should be injected during a polymer flood? Review of previous and current practices, SPE J. 22, 001-018. doi: 10.2118/179543-PA.

Seright R.S., Seheult J.Mac, Talashek T. (2009) Injectivity characteristics of EOR polymers, SPE Reserv. Eval. Eng. 12, 783-792. doi: 10.2118/115142-PA.

Sha F., Xiao L., Mao Z., Jia C. (2019) Petrophysical characterization and fractal analysis of carbonate reservoirs of the eastern margin of the pre-Caspian Basin, Energies 12. doi: $10.3390 /$ en12010078.

Sheng J.J. (2011) Modern chemical enhanced oil recovery, Gulf Professional Publishing.

Sheng J.J., Leonhardt B., Azri N. (2015) Status of PolymerFlooding Technology, J. Can. Pet. Technol. 54, 116-126. doi: 10.2118/174541-PA.

Sorbie K.S. (1991) Polymer-improved oil recovery, Blackie and Son Ltd.

Sorbie K.S. (1990) Depleted layer effects in polymer flow through porous media, J. Colloid Interface Sci. 139, 299314. doi: 10.1016/0021-9797(90)90103-U.

Standnes D.C., Skjevrak I. (2014) Literature review of implemented polymer field projects, J. Pet. Sci. Eng. 122, 761-775. doi: 10.1016/j.petrol.2014.08.024.

Stavland A., Jonsbraten H., Lohne A., Moen A., Giske N.H. (2010) Polymer flooding - flow properties in porous media versus rheological parameters, in: SPE EUROPEC/EAGE Annual Conference and Exhibition, Society of Petroleum Engineers, pp. 14-17. doi: 10.2118/131103-MS.

Taber J.J., Martin F.D. (1983) Technical screening guides for the enhanced recovery of oil, in: SPE Annual Technical Conference and Exhibition, Society of Petroleum Engineers, pp. 1-20. doi: 10.2118/12069-MS.

Taber J.J., Martin F.D., Seright R.S. (1997) EOR screening criteria revisited - Part 1: Introduction to screening criteria and enhanced recovery field projects, SPE Reserv. Eng. 12, 189-198. doi: 10.2118/35385-PA.

Thombare N., Jha U., Mishra S., Siddiqui M.Z. (2016) Guar gum as a promising starting material for diverse applications: A review, Int. J. Biol. Macromol. 88, 361-372. doi: 10.1016/j. ijbiomac.2016.04.001.

Wang D., Cheng J., Xia H., Li Q., Shi J. (2001) Viscous-elastic fluids can mobilize oil remaining after water-flood by force parallel to the oil-water interface, SPE Asia Pacific Improved 
Oil Recovery Conference, Society of Petroleum Engineers. doi: 10.2118/72123-MS.

Wang D., Cheng J., Yang Q., Wenchao G., Qun L., Chen F. (2000) Viscous-elastic polymer can increase microscale displacement efficiency in cores, in: SPE Annual Technical Conference and Exhibition, Society of Petroleum Engineers, pp. 45-51. doi: 10.2118/63227-MS.

Wang D., Wang G., Wu W., Xia H., Yin H. (2007) The influence of viscoelasticity on displacement efficiency - from micro to macro scale, in: SPE Annual Technical Conference and Exhibition. Society of Petroleum Engineers, pp. 1-10. doi: 10.2118/109016-MS.

Wardlaw N.C. (1996) Factors affecting oil recovery from carbonate reservoirs and prediction of recovery, in: G.V. Chilingarian, S.J. Mazzullo, H.H. Rieke (eds.), Carbonate reservoir characterization: A geologic - engineering analysis, Elsevier Science, pp. 867-903.

Wei C., Tian C., Zheng J., Cai K., Du D., Song B., Hu Y. (2015) Heterogeneity characteristics of carbonate reservoirs: A case study using whole core data, SPE Reserv. Characterisation Simul. Conf. Exhib. RCSC 2015, 489-500. doi: 10.2118/ 175670-MS.

Willhite G.P., Dominguez J.G. (1977) Mechanisms of polymer retention in porous media, improved oil recovery by surfactant and polymer flooding, Academic Press, Inc. doi: 10.1016/ B978-0-12-641750-0.50021-9.
Xin X., Xu G., Gong H., Bai Y., Tan Y. (2008) Interaction between sodium oleate and partially hydrolyzed polyacrylamide: A rheological study, Colloids Surfaces A Physicochem. Eng. Asp. 326, 1-9. doi: 10.1016/ j.colsurfa.2008.05.009.

Yanaki T., Kojima T., Norisuye T. (1981) Triple helix of scleroglucan in dilute aqueous sodium hydroxide, Polym. J. 13, 1135-1143. doi: 10.1295/polymj.13.1135.

Zaeri M.R., Hashemi R., Shahverdi H., Sadeghi M. (2018) Enhanced oil recovery from carbonate reservoirs by spontaneous imbibition of low salinity water, Pet. Sci. 15, 564-576. doi: $10.1007 / \mathrm{s} 12182-018-0234-1$.

Zhang G., Seright R.S. (2015) Hydrodynamic retention and rheology of EOR polymers in porous media, Soc. Pet. Eng. SPE 173728, 13-15. doi: 10.2118/173728-MS.

Zhang G., Seright R.S. (2014) Effect of concentration on HPAM retention in porous media, SPE J. 19, 373-380. doi: 10.2118/ 166265-PA.

Zhong K., Liu L., Tong L., Zhong X., Wang Q., Zhou S. (2013) Rheological properties and antitumor activity of schizophyllan produced with solid-state fermentation, Int. J. Biol. Macromol. 62, 13-17. doi: 10.1016/j.ijbiomac.2013.08.015.

Ziauddin M., Bize E. (2007) The effect of pore-scale heterogeneities on carbonate stimulation treatments, in: Proceedings of SPE Middle East Oil and Gas Show and Conference, Society of Petroleum Engineers, pp. 133-146. doi: 10.2523/104627-MS. 